\title{
Inadequacy of 1D, 2D and 3D Resistivity Inverse Modelling in the Presence of Electrical Anisotropy
}

\author{
Branislav Sretenovic, Filip Arnaut \\ University of Belgrade, Faculty of Mining and Geology, Department of Geophysics, Belgrade, Serbia \\ Email address: \\ branislav.sretenovic $a$ rgf.bg.ac.rs (B. Sretenovic), G94-16 $a$ rgf.bg.ac.rs (F. Arnaut) \\ To cite this article: \\ Branislav Sretenovic, Filip Arnaut. Inadequacy of 1D, 2D and 3D Resistivity Inverse Modelling in the Presence of Electrical Anisotropy. \\ Earth Sciences. Vol. 8, No. 2, 2019, pp. 102-116. doi: 10.11648/j.earth.20190802.14
}

Received: February 11, 2019; Accepted: April 9, 2019; Published: April 29, 2019

\begin{abstract}
Universal property of matter is the variation of a certain physical characteristic in different direction. The Substances that do not display this property are an exception. Anisotropy, as this property is named, is also notable in electrical conductivity of minerals, ores, rocks and geological formations. In order to properly define a geological model of an investigated area, it is necessary to account for electrical anisotropy and lateral effects of different origin that are almost always present phenomena. The degree of knowledge of these phenomena determines the quality of interpretation. The effects of electrical anisotropy on 1D, 2D and 3D inversion of apparent resistivity data were examined and the way to detect, quantify and analyse electrical anisotropy in 3D case is proposed. The results of this analysis showed that electrically anisotropic models have led to the totally erroneous results of 3D inversion while the effects of electrical anisotropy on 1D and 2D inversion were less pronounced. Among several existing ways of collecting 3D apparent resistivity data the complete Pole-pole array data set is the only one suitable for detection of electrical anisotropy. Pole-pole resistivity data enable calculating of corresponding resistance or apparent resistivity values for all other collinear or square arrays. This fact makes possible to use standard grid of electrodes in performing 3D Pole-pole apparent resistivity measurements for calculating square array apparent resistivity data. In the special case of vertical stratification (fracturing, schistosity..) this calculated square array data were used for electrical anisotropy analysis thus determining values of coefficient of anisotropy $(\lambda)$ and mean geometric resistivity $\left(\rho_{\mathrm{m}}\right)$. The simple two and three layer 1D synthetic anisotropic models, were used to determine parameters of electrical anisotropy by using 3D forward modelling to calculate Pole-pole and then square array apparent resistivity values. The mean geometric resistivity data obtained by using square array, which are orientation-independent, were used for 1D inversion leading to more realistic results. In the case of oblique stratification (lamination, fracturing, schistosity or karstification) crossed square resistivity data can be reconstructed from 3D Pole-pole data set and then can be used to get parameters of electrical anisotropy, namely apparent coefficient of anisotropy $(n)$, mean geometric resistivity $\left(\rho_{m}\right)$ and apparent electrical strike $(\theta)$. These parameters provide an indication that investigations of electrical anisotropy should be conducted (by using square and crossed square array) in order to avoid erroneous results of 3D inversion.
\end{abstract}

Keywords: Electrical Anisotropy, Oblique Lamination, Rock Fracturing, Schistosity, Coefficient of Anisotropy, Forward Resistivity Modelling, Inverse Resistivity Modelling

\section{Introduction}

Different sedimentological environments (fluvial, lacustrine, glacial, glacio-fluvial, glacio-lacustrine, coastal sand dunes, river dunes, beach ridges, near coastal marine sands, estuarine deposits) lead to depositing sediments characterized by various kinds of oblique lamination which can be resolved by using high resolution ground penetrating radar (GPR) or reflection seismic methods. Electrical DC or low frequency (several Hz) investigation can't resolve such fine lamination inside layers. Various kinds of oblique lamination can cause phenomenon of electrical anisotropy which in return greatly affects $1 \mathrm{D}, 2 \mathrm{D}$ and $3 \mathrm{D}$ resistivity inverse modelling regarding interpreted depths to layer boundaries as well as "true" resistivity of such anisotropic media. The same is the case when dealing with rock fracturing, schistosity or karstification.

Many authors have been dealing with the electrical 
anisotropy caused by fracturing. One can find very comprehensive description of the phenomenon as well as previous developments in the recent paper by

$\mathrm{N}$. Inyang Udosen and N. Jimmy George [1]. These authors performed field electrical resistivity tomography (ERT) investigations along two perpendicular lines and at the crossing of these profiles they conducted azimuthal square array resistivity measurements (changing square array orientation from $0^{\circ}$ to $180^{\circ}$ in increments of $15^{\circ}$ ) and determined electrical anisotropy. To compute the fracture strike analytically, the crossed square array was used [2]. These authors report that: "At small square array spacing of (5 and $7.1 \mathrm{~m}$ ), slight anisotropic responses may be present on the plots but lateral resistivity variations exceed that due to anisotropy. At an electrode spacing of $10 \mathrm{~m}$, the plot became elliptical, indicating anisotropy. These plots became more elliptical as the electrode spacing increased from 10 to $50 \mathrm{~m}$. The anisotropic coefficients of the limestone layer were found to range from $1.37 \pm 0.075$ to $1.44 \pm 0.075$, indicating moderate anisotropy". They also report that: "ERT data was acquired to generate a 2D image of the subsurface, determine depth to bedrock, and ascertain the lithological variations within the subsurface." These goals are hard to achieve in the presence of electrical anisotropy by using ERT data. Although these authors claim that "Ignoring the effects of electrical anisotropy within the subsurface while taking field measurements gives unsatisfactory results" [1], they do ignore the effects of electrical anisotropy on 2D inversion of ERT data claiming that "The inversion model was considered reasonably correct due to the small root mean square error of $0.67 \%$ ". Electrical anisotropy probably affected the results of 2D inversion of ERT data along the two perpendicular profiles but authors didn't take it in consideration. The aim of this paper is to show how inadequate $1 \mathrm{D}, 2 \mathrm{D}$ or $3 \mathrm{D}$ resistivity inversion can be in the presence of electrical anisotropy but also to suggest the possibility of detecting and quantifying electrical anisotropy in 3D case.

Colossal advance in the field of DC resistivity method took place in the last thirty years, regarding instrument improvements, automatization of data acquisition and especially in forward and inverse modelling techniques. All improvements that had been achieved allows us to try to improve limited resolving power of DC resistivity method by analysing electrical anisotropy and by using its elements, namely the mean geometric apparent resistivity $\left(\rho_{\mathrm{m}}\right)$, apparent coefficient of anisotropy (n) and apparent electrical strike $(\theta)$.

Bhattacharya and Patra [3] solved analytically problem of a potential distribution over homogeneous anisotropic subsurface model (Figure 1). Model is made under the assumption that the plane of stratification, lamination or bedding is tilted by an angle $(\alpha)$ in respect to the surface and $(\phi)$ is the angle between layering strike and current electrodes (in the case of square array).

Property of a homogeneous anisotropic subsurface is dependence of measured resistivity upon the direction. Value of true resistivity parallel to the plane of stratification $\left(\rho_{1}\right)$ is smaller than the value perpendicular to that plane $\left(\rho_{t}\right)$. These two values determine coefficient of anisotropy $\lambda=\sqrt{ } \rho \mathrm{t} / \rho \mathrm{l}>1$. On the other hand, measured apparent resistivity varies with array orientation to the electrical strike, being minimal when the orientation is perpendicular to it and maximal in the case of parallel array orientation. This is well known paradox of anisotropy which is characteristic of all collinear arrays.

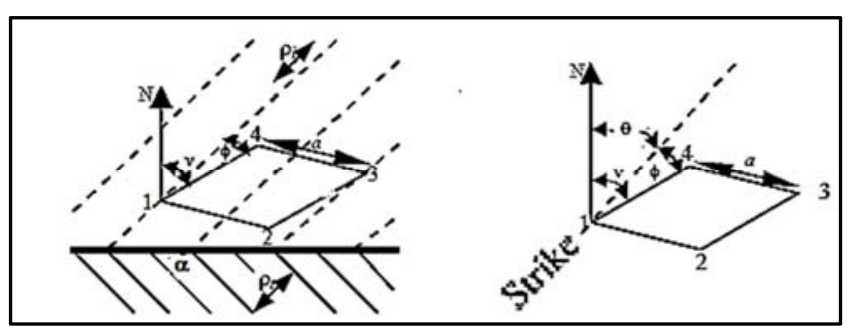

Figure 1. Homogeneous anisotropic subsurface model [3].

For modelling purposes software created by M.H. Loke [46] as well as 1D resistivity sounding inversion software created by Vander Velpen [7 ] were used. The three layer 1D anisotropic model was created by using 2D forward modelling (RES2DMOD). For this three layer 1D model, with the highly anisotropic middle layer, the Wenner apparent resistivity cross-section was calculated. The calculated values were used to reconstruct Wenner alpha sounding curve and then to use it for 1D inversion. There is an excellent option offered by RES2DMOD software to save calculated apparent resistivity data in the format used by inversion RES2DINV software and to simulate measured data by adding a certain level of random noise to the calculated data ( $\pm 2 \%$ in this case). Theoretically calculated data with $\pm 2 \%$ random noise added to it was then inverted by using RES2DINV software and as a result an inverse model resistivity section was created. The same approach of combining forward and inverse modelling was applied for more complex 2D anisotropic models as well as for $3 \mathrm{D}$ anisotropic models.

\section{The Results of Analysing Anisotropic Models}

\subsection{One-Dimensional Anisotropic Models}

Figure 2 (lower part) shows 1D anisotropic three layer model, the middle layer being highly anisotropic, while the relatively thin covering layer $(2.5 \mathrm{~m}, 50 \mathrm{Ohmm})$ and the third layer (3000 Ohmm) are homogenous and isotropic. The middle layer consists of steeply dipping thin layers with alternating true resistivity values of 300 and 10 Ohmm respectively, which gives rise to strong electrical anisotropy. This anisotropy is detectable by resistivity measurements conducted from the surface due to inclination of alternating high and low resistivity thin layers.

The upper part of Figure 2 shows the calculated Wenner array apparent resistivity cross-section which indicates horizontal layering where the middle layer (blue zone of the 
section) appears as an isotropic and relatively low resistive (28-32 Ohmm) layer. The third high resistivity layer (purple zone of the section) is indicated by maximum apparent resistivity values of about $95 \mathrm{Ohmm}$. After 2D inversion of this apparent resistivity cross-section the 2D inverse model resistivity section is obtained with RMS error of $1.88 \%$ (Figure 3). The middle anisotropic layer is characterized by even lower "true" resistivity value (16.5-27.5 Ohmm) and it appears to be an isotropic layer. Two-dimensional inversion clearly indicates presence of the covering layer (with the resistivity of 45-50 Ohmm) and high resistivity substratum (over $650 \mathrm{Ohmm}$ ). The depth to the third layer (indicated by solid red line) is underestimated by at least $15-20 \%$ due to the fact that the middle layer is anisotropic. Furthermore there is no indication of electrical anisotropy of the second layer. In the case of horizontal stratification or lamination, anisotropy is not detectable by using measurements conducted from the surface and interpreted depth to substratum would be overestimated by the factor equal to the coefficient of anisotropy $\lambda=\sqrt{ } \rho_{\mathrm{t}} \quad / \rho_{\mathrm{p}}>1$ [8]. Inclined stratification or lamination leads to apparent coefficient of anisotropy (n) which is always smaller than $(\lambda)$, except in the case of vertical stratification when it is equal to $(\lambda)$.

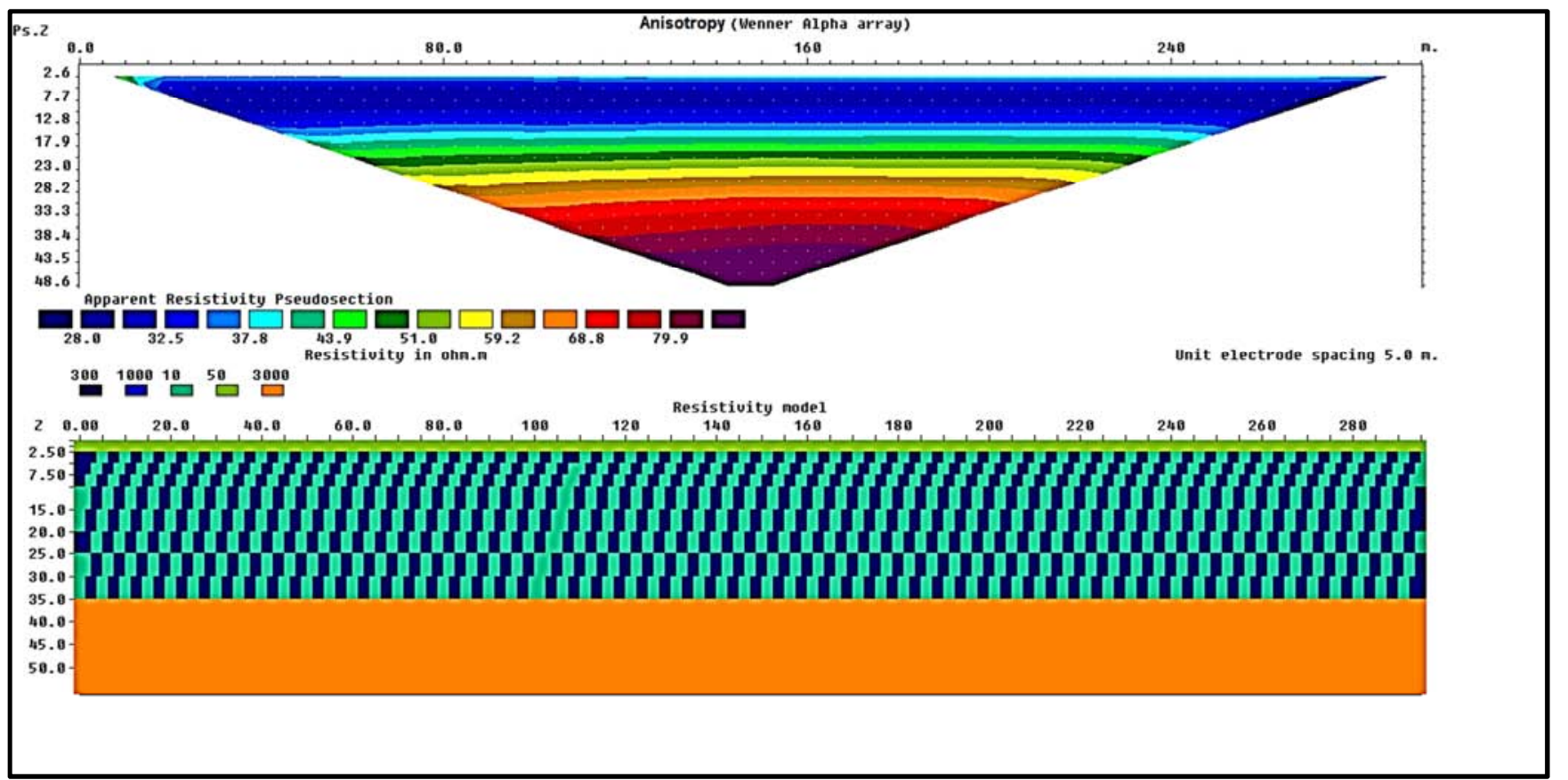

Figure 2. 1D anisotropic three layer model and corresponding apparent resistivity cross-section.

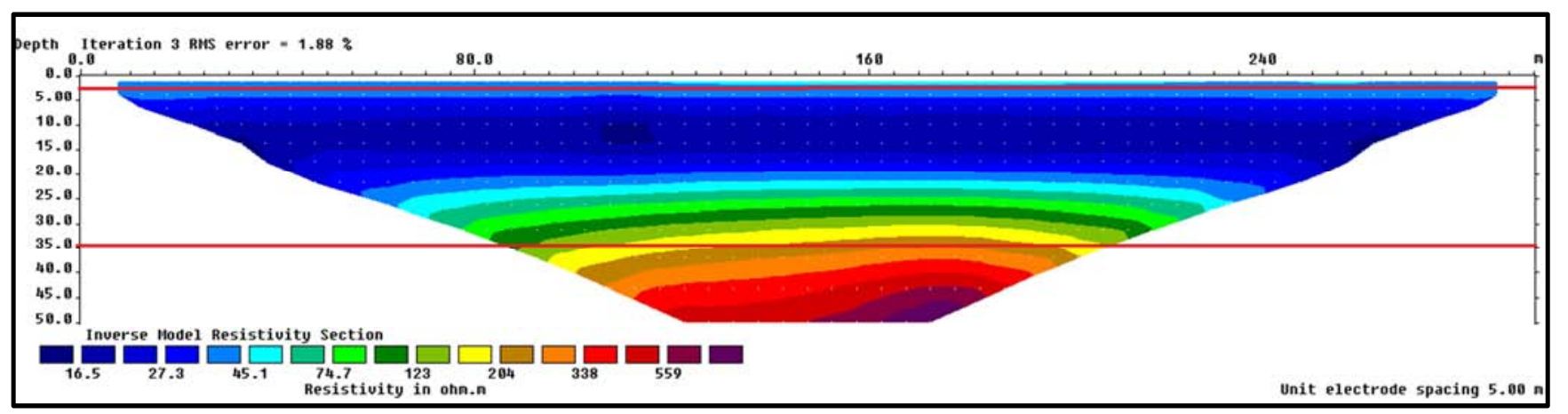

Figure 3. $2 D$ Inverse model resistivity section.

Two-dimensional modelling was performed by using Wenner alpha array oriented perpendicularly to the strike of thin inclined layers (stratification or lamination) inside the middle layer in the 1D anisotropic model. Measured apparent resistivity varies with array orientation to the electrical strike, being minimal when the orientation is perpendicular to it and maximal in the case of parallel array orientation. Therefore one could expect higher values of apparent resistivity with array orientations other than perpendicular and consequently different results of 2D inversion.

In order to determine "true" resistivity of the middle layer it is possible to perform $1 \mathrm{D}$ interpretation by fixing the values of all parameters known from the model (i.e. thicknesses of layers and resistivity of the covering layer and the third layer). Figure 4 shows that the value of root mean square error is of an order defined by the level of random noise added to the theoretically calculated data (RMS Error $=2.1 \%$ ) and that the resistivity of the middle layer is 28.5 
Ohmm. It is clearly visible that theoretically calculated resistivity sounding curve (---) deviates the most from the measured data $(++)$ in the zone of curve minimum which corresponds to the middle layer. Figure 5 shows the results of 1D interpretation with lower value of the second layer resistivity (25 Ohmm). Keeping the RMS error value around $2 \%$ and not allowing changing of the parameters of the first and the third layer, further lowering of the second layer resistivity was not possible. Thickness of the second layer was reduced from 32.5 meters to about 27 meters and the depth to substratum from 35 meters to 29.4 meters, which is closer to the results of 2D inversion in Figure 3. The range of equivalence in this case was very narrow since the H-type apparent resistivity sounding curve which corresponds to the 1D model almost reaches the "true" resistivity of the middle layer (the second to the first layer thickness ratio being 13:1).

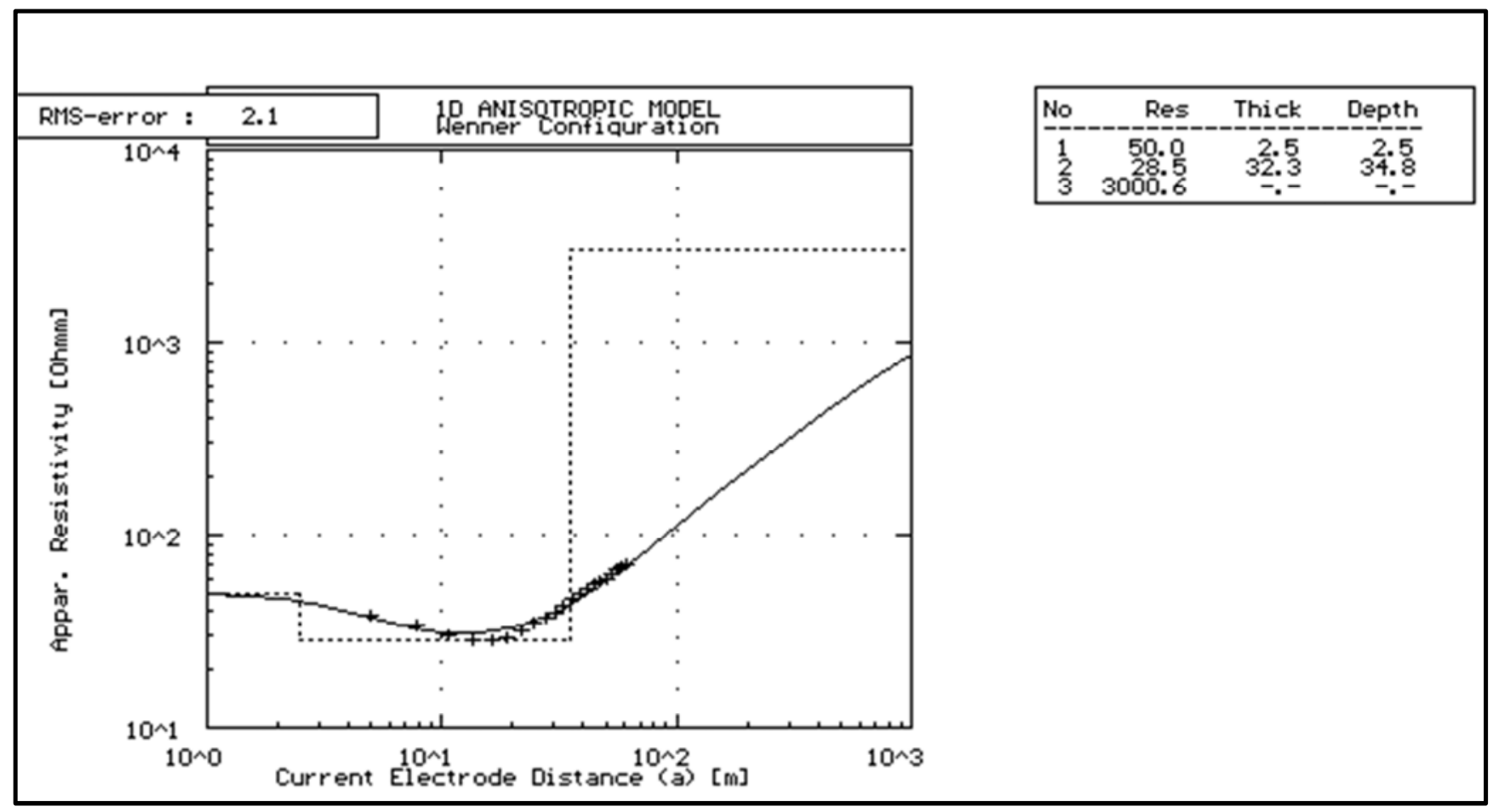

Figure 4. $1 D$ interpretation of Wenner sounding data over the three layer1D anisotropic model.

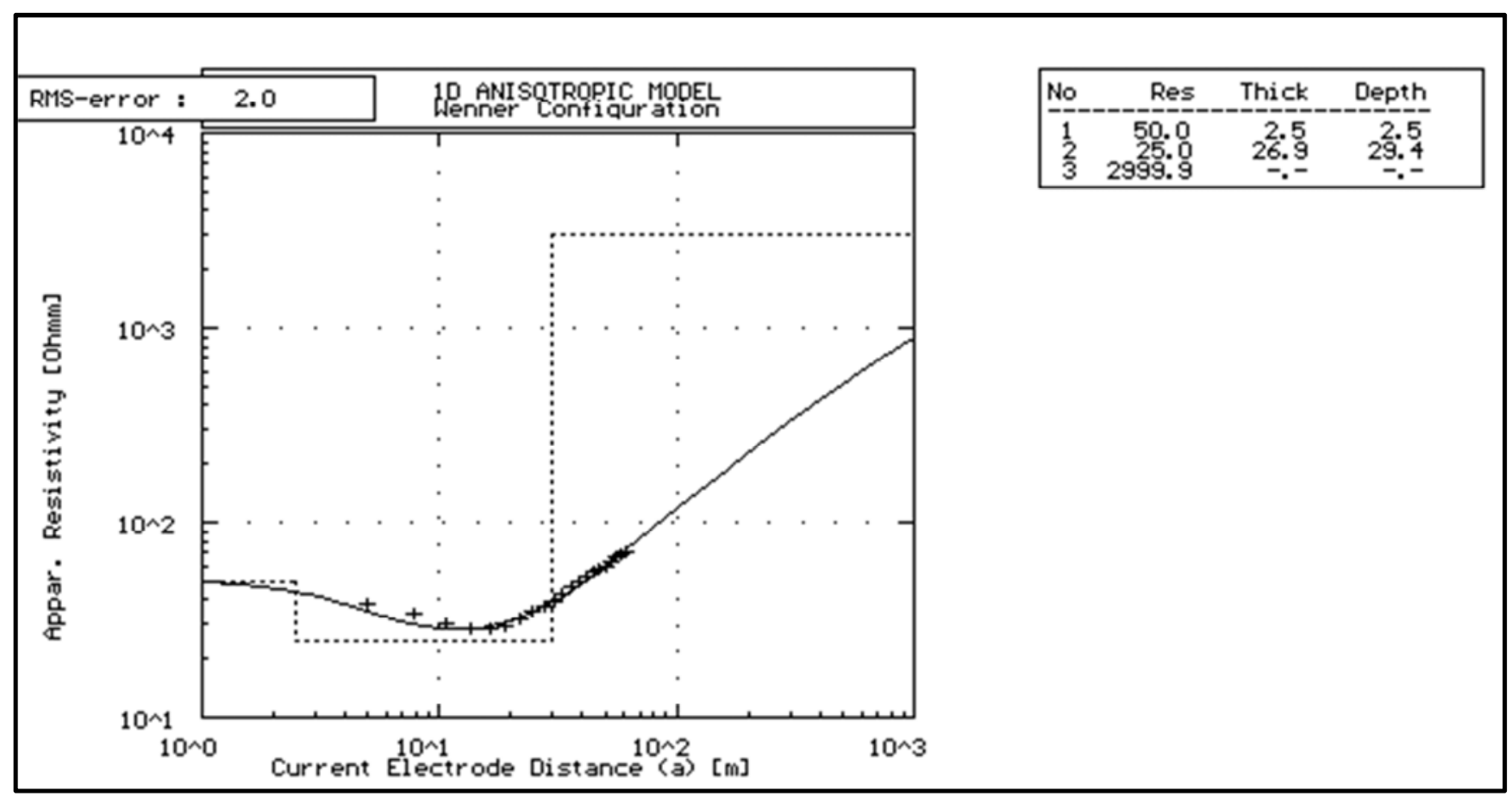

Figure 5. $1 D$ equivalent interpretation of Wenner sounding data over the three layer $1 D$ anisotropic model. 


\subsection{Two-Dimensional Anisotropic Models}

The two-dimensional anisotropic model consists of the covering layer $(5 \mathrm{~m}, 50 \mathrm{Ohmm})$, anisotropic middle layer with the same internal structure as in the case of 1D anisotropic model (resistivity contrast between thin inclined layers amounts 30:1) and high resistivity substratum (3000 Ohmm) in the form of trench structure dipping from 35 to 55 meters in the middle of profile (Figure 6 - lower part). Apparent resistivity cross-section (Figure 6 - upper part) was calculated for Wenner alpha array with 60 electrodes and unit electrode spacing of 5 meters. It reflects the presence of the trench structure and low resistivity zone (30-35 Ohmm) which corresponds to the middle anisotropic layer. Two-dimensional inversion was carried out by using calculated apparent resistivity data set with $\pm 2 \%$ random noise level added to it, so that RMS error of $2.5 \%$ is quite satisfactory. The result of the $2 \mathrm{D}$ inversion reveals the fact that anisotropic layer is characterized by even lower "true" resistivity value (14-23 Ohmm) and with underestimated depth to substratum of about $15-20 \%$, while general trench-like structure is well defined. Red solid lines in Figure 7 depict the true depths to boundaries in the $2 \mathrm{D}$ model.

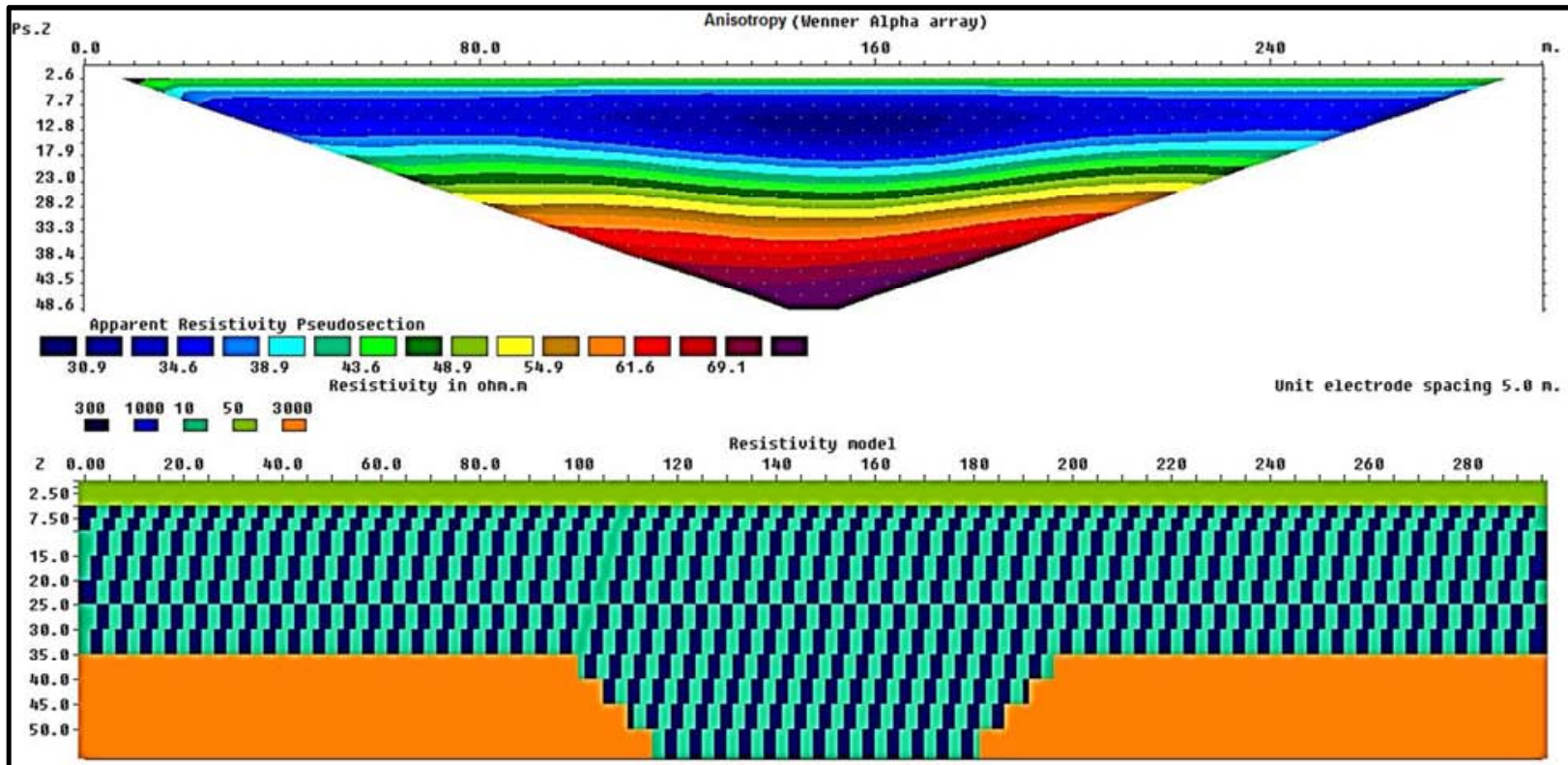

Figure 6. 2D anisotropic model and Wenner apparent resistivity cross-section

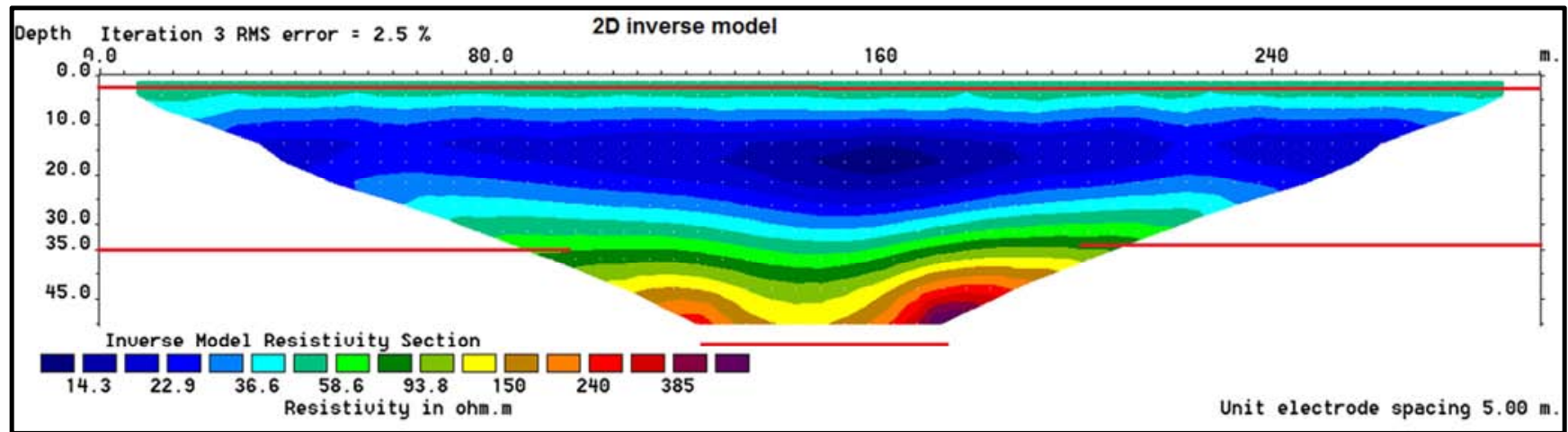

Figure 7.2D inverse model resistivity section.

In order to investigate 3D forward and inverse modelling ten parallel 2D Wenner array profiles were created with the same anisotropic layer present and by using the same covering layer and substratum parameters. The depth to the substratum and to the bottom of the trench is getting shallower from profile 1 towards profile 10, while the trench structure is getting narrower. Figure 8 shows three out of ten parallel profiles (Profiles 1, 6 and 10). These ten calculated $2 \mathrm{D}$ apparent resistivity data sets with $\pm 2 \%$ random noise level added to it were initially inverted independently to give a series of $2 \mathrm{D}$ cross-sections, the three of them are given in Figure 9.

"The measured apparent resistivity values from all the lines can also be combined into a $3 \mathrm{D}$ data set and inverted with 
RES3DINV to give a 3D picture. While the quality of the $3 \mathrm{D}$ model is expected to be poorer than that produced with a complete 3D survey, such 3D data set could reveal major resistivity variations across the survey lines. While it is possible to combine a number of $2 \mathrm{D}$ lines into a $3 \mathrm{D}$ data set, it might not always be worthwhile to do so. Firstly, it is recommended that there should be at least 5 parallel lines. Furthermore, the separation between the lines should not be more than twice the unit electrode spacing along the lines" [4].

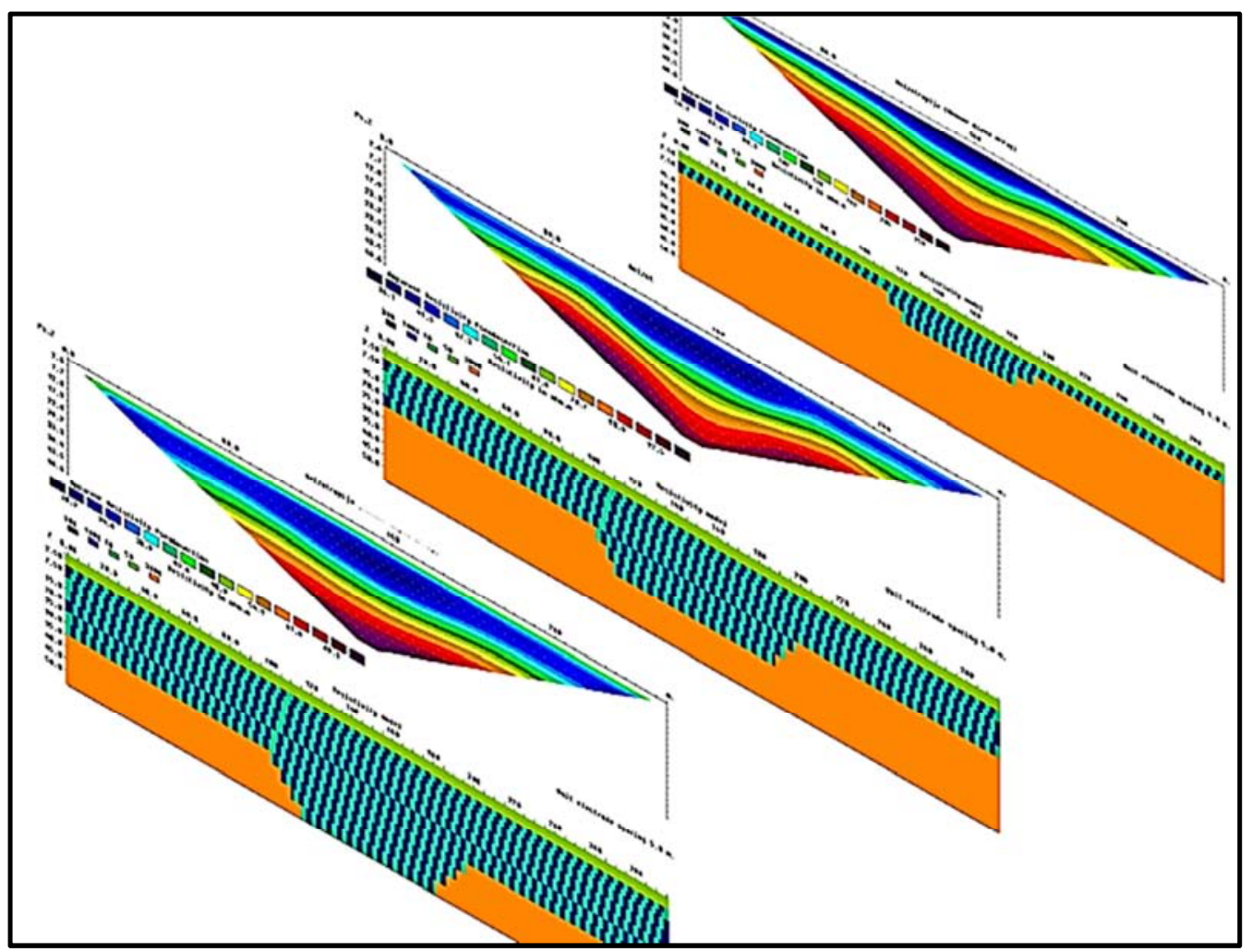

Figure 8. Three parallel 2D profiles together with corresponding Wenner apparent resistivity cross- sections.

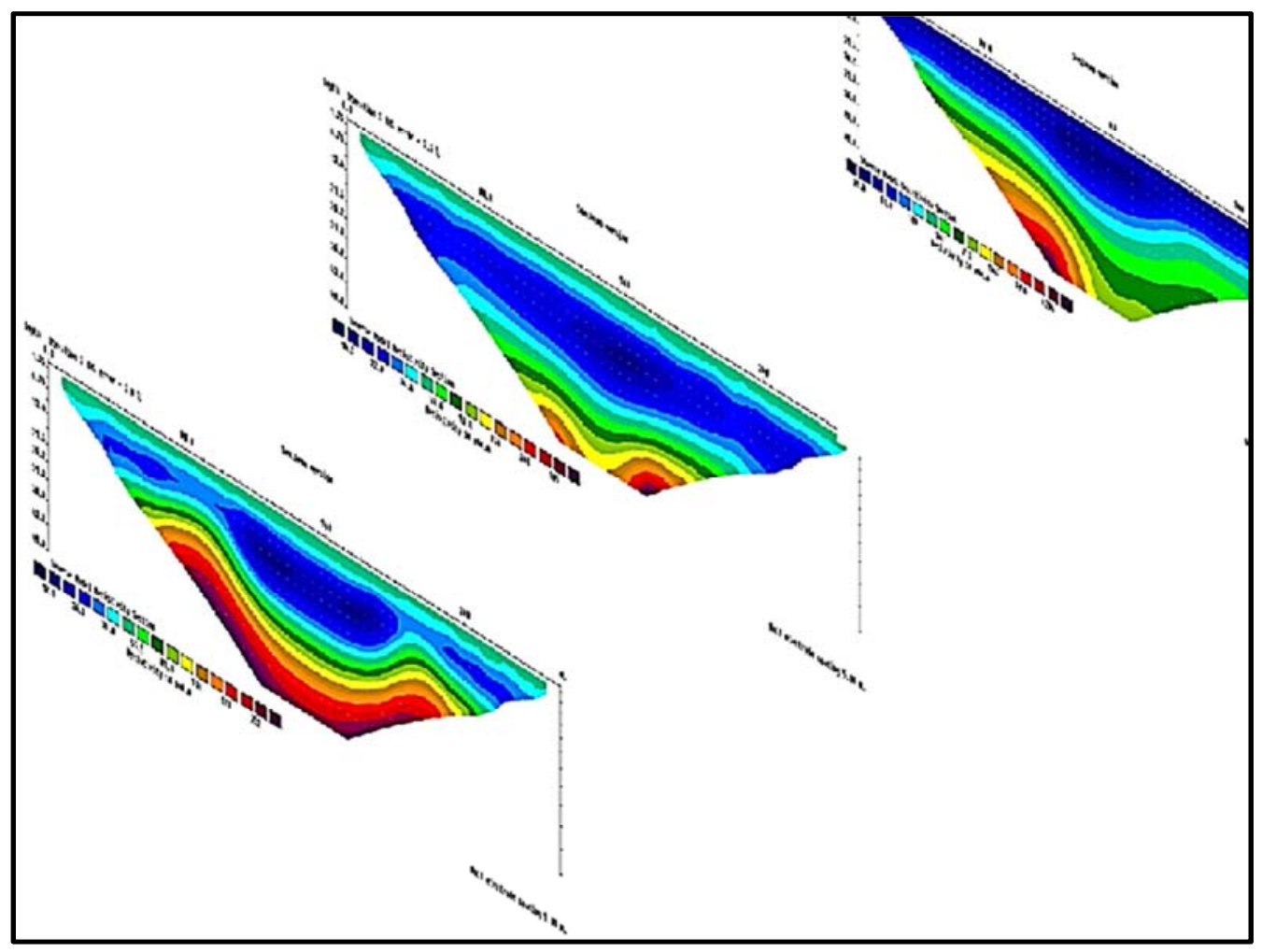

Figure 9. The three out of ten $2 D$ inversion models along parallel profiles. 


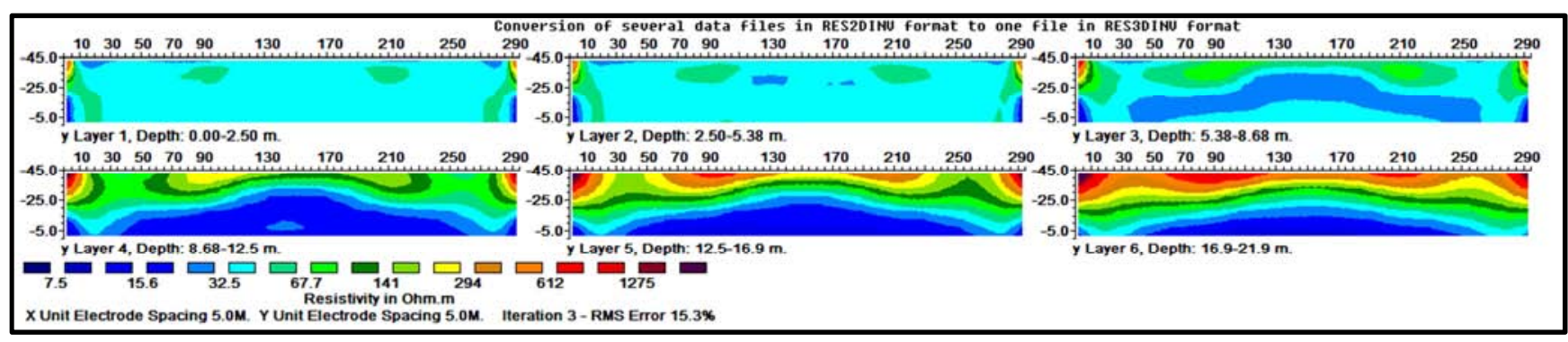

Figure 10. 3D inversion based on the ten inverted $2 D$ apparent resistivity data sets $(Y: X$ axes $=2: 1)$.

Three-dimensional inversion based on 2D apparent resistivity data sets along ten parallel profiles (5 meters apart) revealed that the anisotropic layer filling up the $2 \mathrm{D}$ trench structure shows very low "true" resistivity values ranging from 7.5 to $15 \mathrm{Ohmm}$, which is much lower than its values based on $1 \mathrm{D}$ and 2D inversions (Figure 10). The covering layer (5meters thick and with resistivity of $50 \mathrm{Ohmm}$ ) is well defined as well as the trench structure which is getting wider and deeper from profile 10 towards profile 1 . True resistivity of substratum is very well determined (2550 Ohmm compared to about $600 \mathrm{Ohmm}$ in the $2 \mathrm{D}$ resistivity inverse model in Figure 7). The results of 3D inversion are presented with six layers with the maximum depth of about 22 meters which is relatively shallow compared to 50 meters in $2 \mathrm{D}$ anisotropic model. Root mean square error which is achieved in just three iterations is $15.3 \%$

\subsection{Three-dimensional Anisotropic Models}

"For relatively small grids of less than 12 by 12 electrodes, the pole-pole array has a substantially larger number of possible independent measurements compared to other arrays. The loss of data points near the sides of the grid is kept to a minimum, and it provides better horizontal data coverage compared to other arrays. This is an attractive array for small survey grids with relatively small spacing (less than
5 meters) between the electrodes. However, it has the disadvantage of requiring two "remote" electrodes that must be placed at a sufficiently large distance from the survey grid. Due to the large distance between the two potential electrodes, this array is more sensitive to telluric noise" [4].

Pole-pole array was used not just for the advantages quoted from [4] but for an additional advantage of Pole-pole array which isn't mentioned by the author [4]. Namely, resistance measurements conducted by using this array make it possible to calculate the corresponding resistance values of other arrays [9] among which the most interesting for the purposes of this paper is square array.

For 1D anisotropic two-layer model (Figure 11) the apparent resistivity $3 \mathrm{D}$ data set was calculated and random noise of $\pm 2 \%$ was added to simulate Pole-pole measured data set. Figures 12 and 13 confirm paradox of anisotropy since apparent resistivity values measured in x-direction, perpendicularly to the strike of vertical thin layers with alternating resistivity values (10 and $300 \mathrm{Ohmm}$ ), are of lower magnitude than with Pole-pole array orientation parallel to the electrical strike (y-direction). Both sets of Pole-pole apparent resistivity cross-sections (X and $\mathrm{Y}$ orientated) show serious deviation from the 1D anisotropic model, since they indicate shallowing of the lowest resistivity values towards surface.

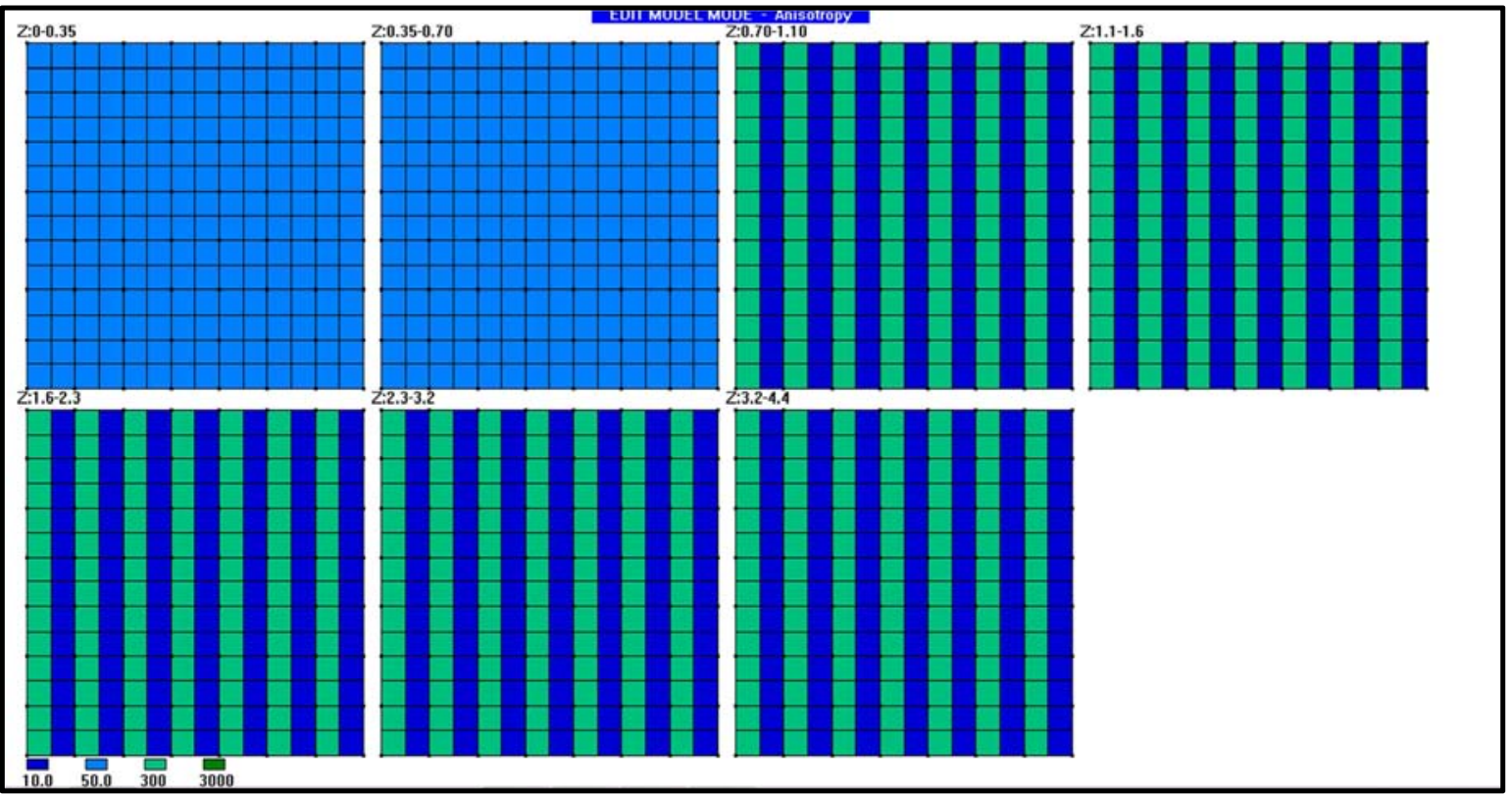

Figure 11. Two layer1D anisotropic model. 


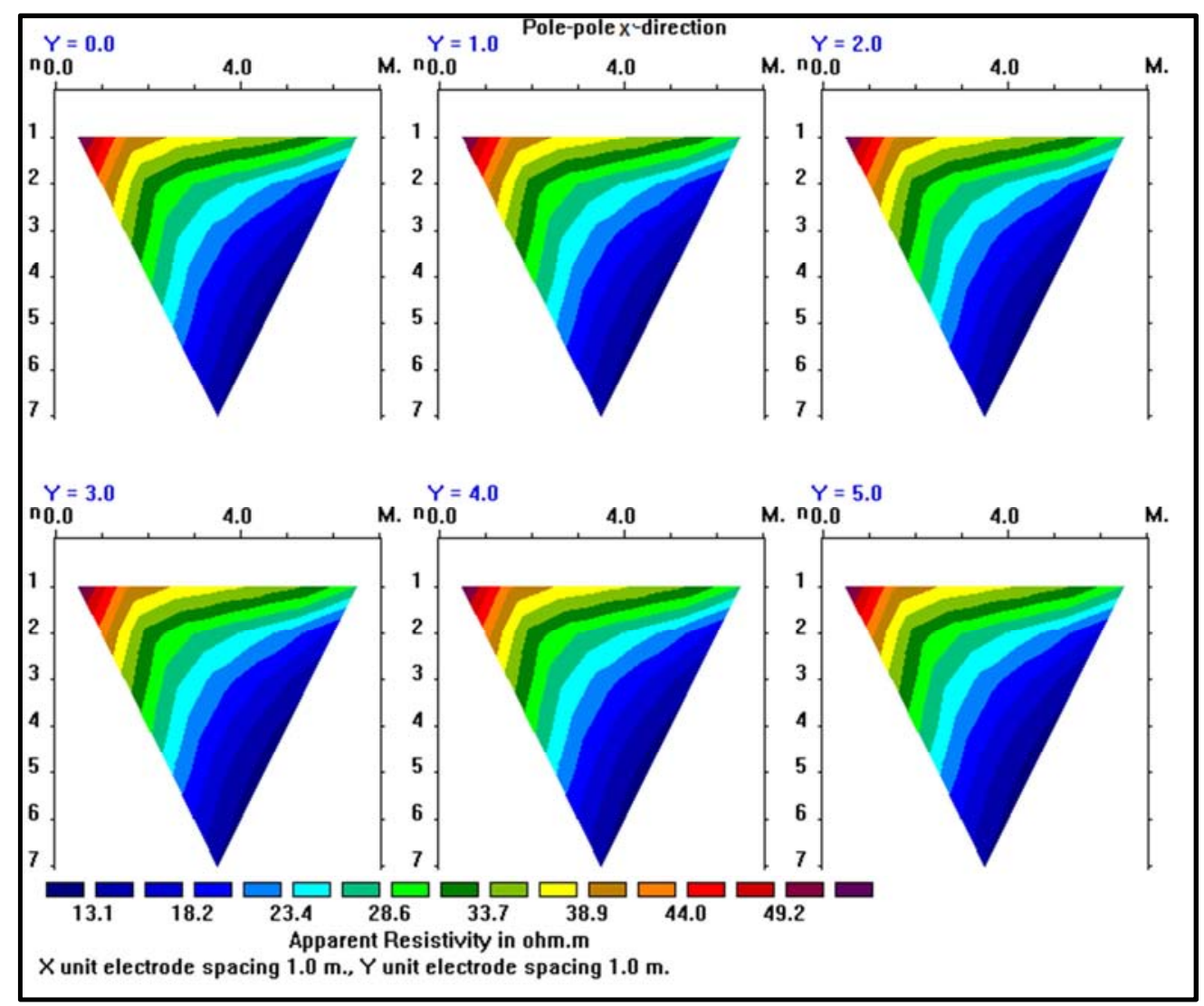

Figure 12. Pole-pole array apparent resistivity cross-sections - X direction.

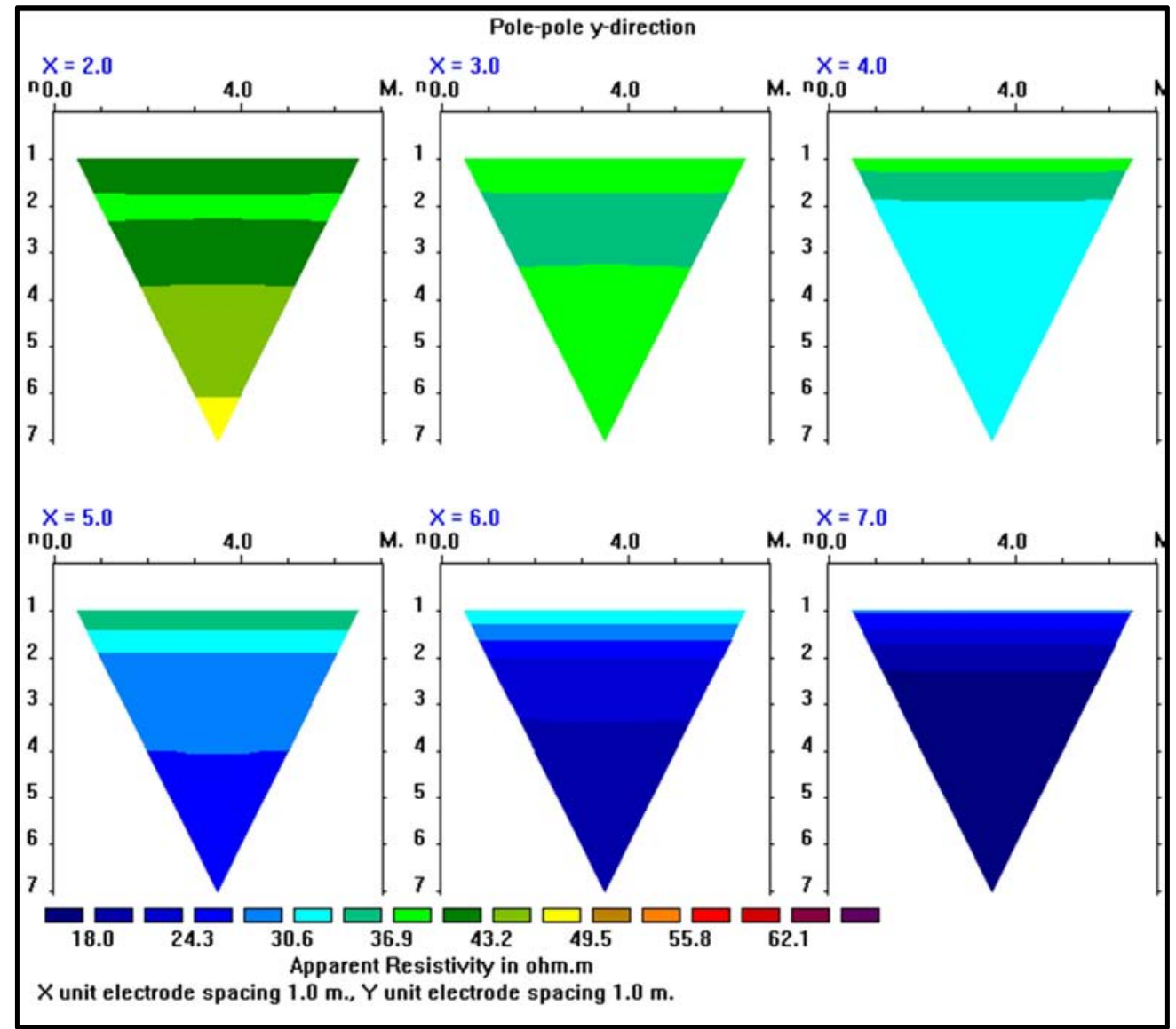

Figure 13. Pole-pole array apparent resistivity cross-sections - $Y$ direction. 


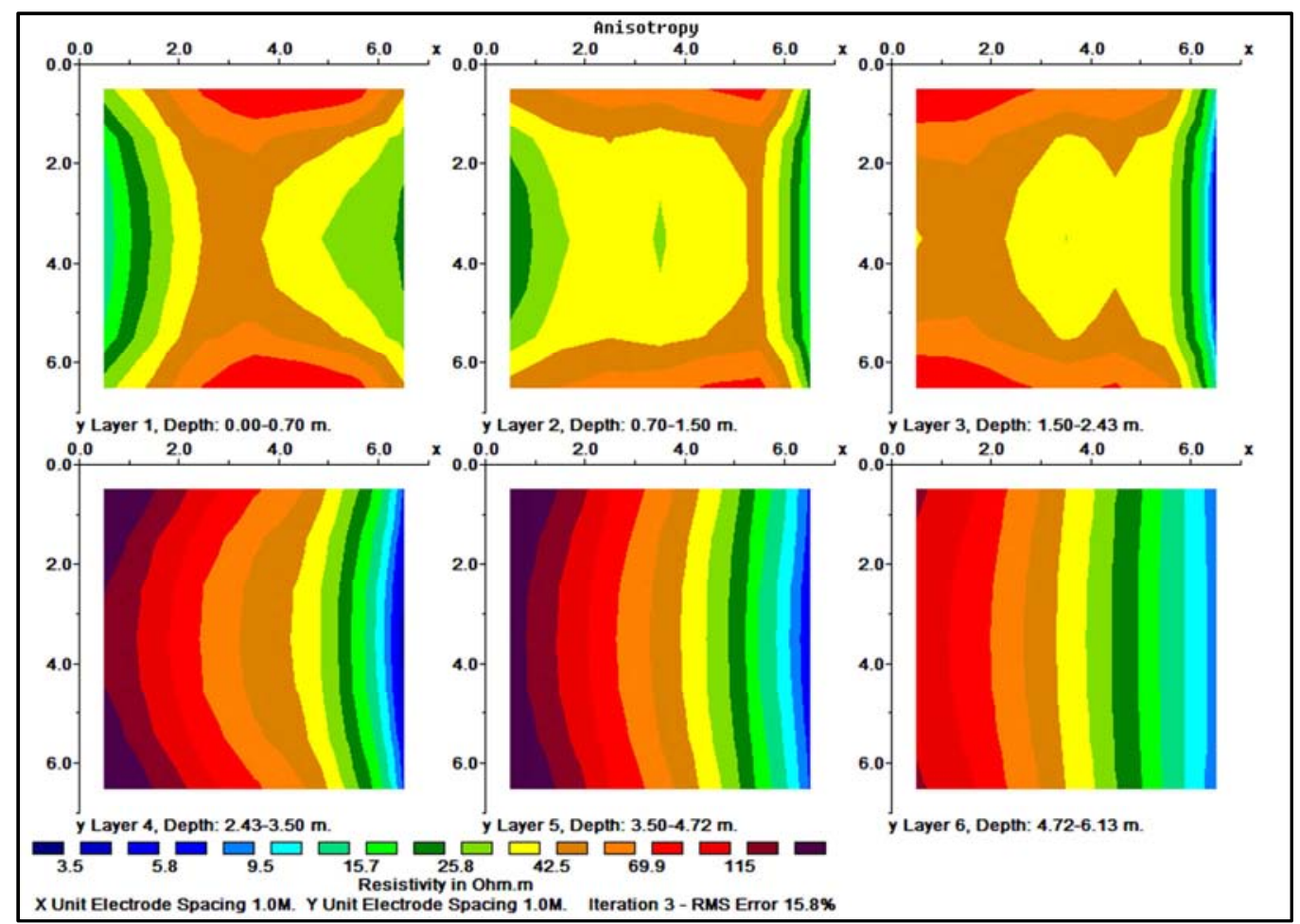

Figure 14. The results of $3 D$ inversion-horizontal sections.

The results of 3D inversion of Pole-pole apparent resistivity complete data set (Figures 14 and 15) are totally erroneous having in mind very simple 1D anisotropic model which was used for this analysis ( Figure 11).

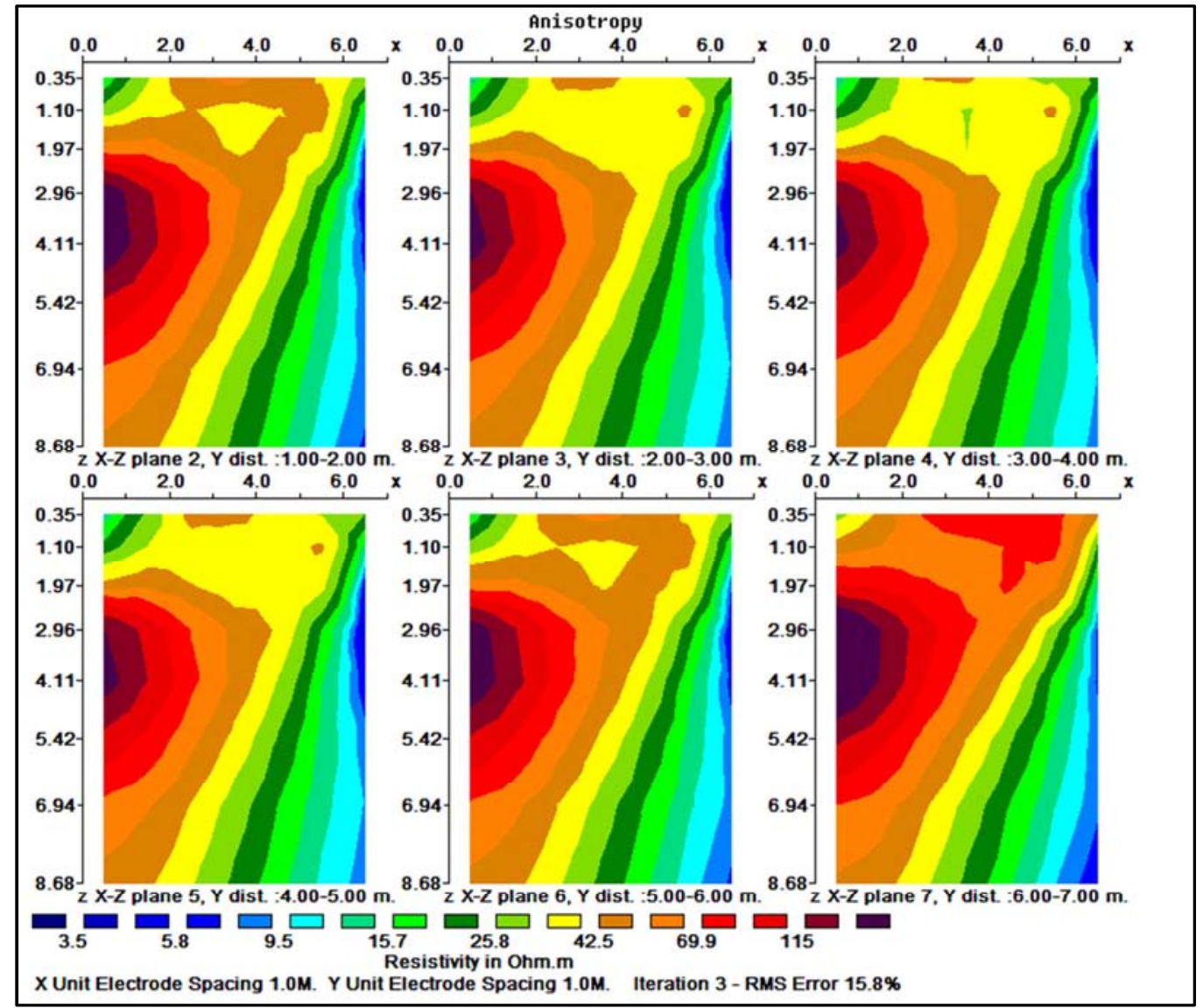

Figure 15. The results of $3 D$ inversion-vertical sections. 


\section{Reconstructing of Square Array Apparent Resistivity Data from the Complete 3D Pole-Pole Measurements}

\subsection{Square and Crossed Square Array}

Investigation of electrical anisotropy by using square array has many advantages compared to investigation conducted with collinear arrays. It requires less surface area than collinear arrays of similar depth of investigation. This array is less sensitive to lateral effects of local heterogeneities, and much more sensitive to presence of electrical anisotropy then collinear arrays [10]. Also, square array doesn't produce paradox of anisotropy. The location of a measurement is assigned to the central point of the square and the array size (a) is defined by the length of the square side. The array is expanded symmetrically about the center point, so that a resistivity sounding curve can be interpreted as a function of depth [11].

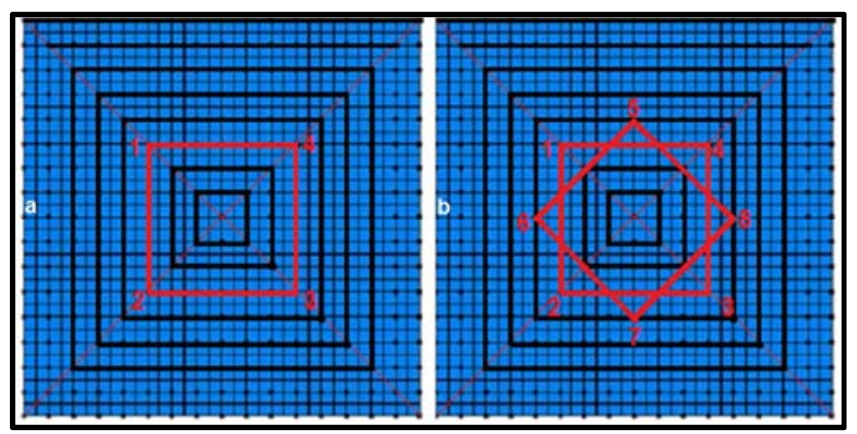

Figure 16. (a) Square array (b) crossed square array.

Since 1D, 2D and 3D inversions of apparent resistivity data sets acquired over an anisotropic half-space lead to erroneous results and give no indication of electrical anisotropy there is a need to reconstruct square array resistivity data from Pole-pole 3D measured apparent resistivity data. Figure 16a illustrates this possibility showing a number of expanding square arrays by using electrodes in a grid (not mesh lines between electrodes). Already existing Pole-pole data in 3D calculated (measured) data set will be used to calculate square array data sets. In order to calculate square array apparent resistivity value $\left(\rho_{\alpha}\right)$ it is possible to use four Pole-pole apparent resistivity measurements (Figure $17 \mathrm{a}$ - yellow and white lines) by summing the four values and finding the average value of that sum. The same procedure is applied for calculating $\left(\rho_{\beta}\right)$ in (Figure 17b). In order to calculate square array resistance values $\left(\mathrm{R}_{\alpha}\right)$ and $\left(R_{\beta}\right),\left(\rho_{\alpha}\right)$ and $\left(\rho_{\beta}\right)$ are divided by appropriate square array geometric factor, $K=2 \pi \mathrm{a} /(2-\sqrt{2})$. The same procedure is applied for calculating $\left(\mathrm{R}_{\alpha}^{\prime}\right)$ and $\left(\mathrm{R}_{\beta}{ }_{\beta}\right)$ for square array rotated by $45^{\circ}$ (Figure $16 \mathrm{~b}$ ) and thus one gets the complete input data for crossed square anisotropy analysis proposed by Habberjam [9-10]. Apparent electrical strike $(\theta)$, apparent coefficient of electrical anisotropy $(n)$ and mean geometric apparent resistivity $\left(\rho_{\mathrm{m}}\right)$ are calculated from electrical resistance $\left(R_{\alpha}, R_{\beta}, R_{\alpha}^{\prime}, R_{\beta}^{\prime}\right)$ in two square orientations, under the assumption of homogeneous anisotropic half-space. Accuracy of determination of axes of anisotropy is no longer dependent on number of array directions, but on disturbances caused by heterogeneities which lead to deviation from homogeneous and anisotropic subsurface model. This inevitable geological noise may be quantitatively accounted for, by using parameter $(\xi)$ which defines the deviation from homogeneous anisotropic subsurface model. Since three unknown parameters $\left(n, \theta, \rho_{m}\right)$ have to be determined, it is enough to consider values of resistance measured in three directions, and the fourth measurement enables determination of parameter $(\xi)$. Orientation of the system is based on the direction defined by the electrodes $1-4$, and the input values are electrical resistances $R_{\alpha}, R_{\beta}, R_{\alpha}{ }_{\alpha}, R^{\prime}{ }_{\beta}$. There is, however, a slight difference of about $5 \%$ in square array side (a) in two different square array orientation (Figure 16b) so the depth of investigation, could be slightly erroneous. Therefore the procedure for anisotropy analysis based on crossed square resistivity measurements will not be used in this paper.

Figure 18 shows the part of 3D (*.dat ) file with coordinates of current electrodes $(\mathrm{Xc}, \mathrm{Yc})$ and potential electrodes $(\mathrm{Xp}, \mathrm{Yp})$ of different Pole-pole configurations which are involved in calculated 3D data set.

The very simple $1 \mathrm{D}$ two-layer anisotropic model was used (Figure 11), with the thin covering isotropic layer $(0.7 \mathrm{~m}, 50$ Ohmm) and anisotropic half-space consisting of thin $(0.5 \mathrm{~m})$ vertical layers with alternating resistivity values (10 and 300 Ohmm). This vertical layering enables determination of anisotropy coefficient $(\lambda)$ with square array resistivity measurements from the surface. Square array was favourable oriented to the electrical strike, so that $\left(\rho_{\alpha}\right)$ value represents apparent resistivity measured perpendicular to electrical strike and $\left(\rho_{\beta}\right)$ apparent resistivity measured parallel to the strike of thin vertical layers which cause electrical anisotropy (Figure 17). The four Pole-pole arrays and corresponding apparent resistivities which can be used for calculating the square array apparent resistivity $\left(\rho_{\alpha}\right)$ values are marked in Figure 18 (red rectangles).

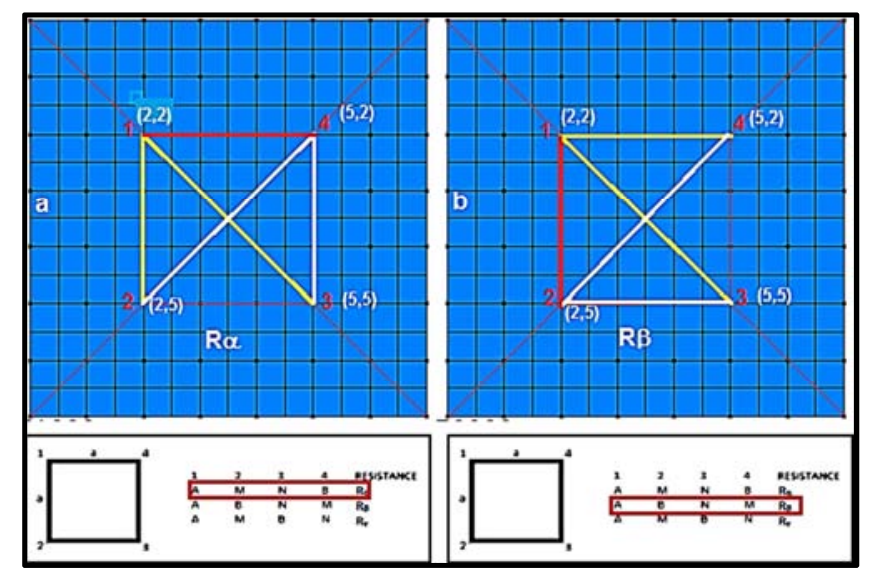

Figure 17. Square array measurements and Pole-pole configurations (a) perpendicular square array orientation (b) parallel square array orientation $[9,10]$. 


$\left.\begin{array}{|llllc||ccccc|}\hline \text { Xc } & Y c & \text { Xp } & Y p & \rho a \\ 2.000 & 2.000 & 1.000 & 4.000 & 40.554 \\ 2.000 & 2.000 & 2.000 & 4.000 & 39.239 \\ 2.000 & 2.000 & 3.000 & 4.000 & 35.025 \\ 2.000 & 2.000 & 4.000 & 4.000 & 28.486 \\ 2.000 & 2.000 & 5.000 & 4.000 & 23.294 \\ 2.000 & 2.000 & 6.000 & 4.000 & 17.404 \\ 2.000 & 2.000 & 7.000 & 4.000 & 12.337 \\ 2.000 & 2.000 & 0.000 & 5.000 & 46.893 \\ 2.000 & 2.000 & 1.000 & 5.000 & 43.708 \\ 2.000 & 2.000 & 2.000 & 5.000 & 42.862 \\ 2.000 & 2.000 & 3.000 & 5.000 & 36.986 \\ 2.000 & 2.000 & 4.000 & 5.000 & 30.640 \\ 5.000 & 2.000 & 5.000 & 5.000 & 23.724\end{array}\right]\left[\begin{array}{ccccc}5.000 & 2.000 & 2.000 & 4.000 & 23.437 \\ 5.000 & 2.000 & 3.000 & 4.000 & 24.635 \\ 5.000 & 2.000 & 4.000 & 4.000 & 28.028 \\ 5.000 & 2.000 & 5.000 & 4.000 & 30.525 \\ 5.000 & 2.000 & 6.000 & 4.000 & 23.747 \\ 5.000 & 2.000 & 7.000 & 4.000 & 15.312 \\ 5.000 & 2.000 & 0.000 & 5.000 & 24.752 \\ 5.000 & 2.000 & 1.000 & 5.000 & 24.074 \\ 5.000 & 2.000 & 2.000 & 5.000 & 24.070 \\ 5.000 & 2.000 & 3.000 & 5.000 & 26.171 \\ 5.000 & 2.000 & 4.000 & 5.000 & 27.819 \\ 5.000 & 2.000 & 5.000 & 5.000 & 28.531 \\ \hline 5\end{array}\right.$

Figure 18. The four Pole-pole apparent resistivity values extracted from $3 D$

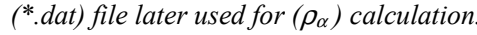

By averaging the sum of the four Pole-pole apparent resistivity data, one gets square array apparent resistivity value $\rho_{\alpha}=29.8 \mathrm{Ohmm}$, with the array orientation perpendicular to the strike of $1 \mathrm{D}$ anisotropic model. On the other hand, by averaging the sum of four Pole-pole apparent resistivity data in Figure 19, one gets square array apparent resistivity value $\rho_{\beta}=23.0 \mathrm{Ohmm}$, with the array orientation parallel to the strike of anisotropic model. It is obvious that there is no paradox of anisotropy for square array.

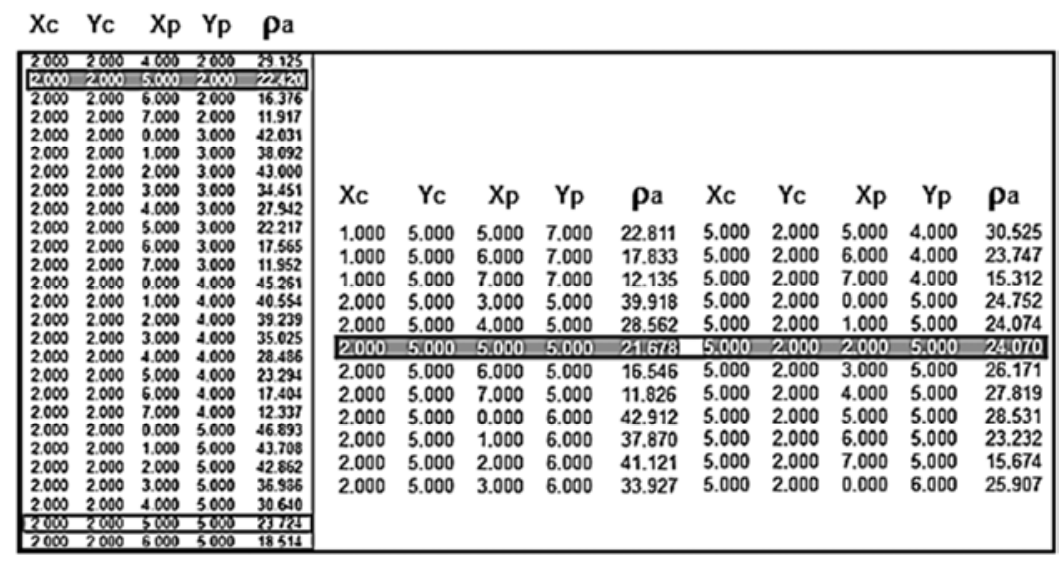

Figure 19. The four Pole-pole apparent resistivity values extracted from $3 D\left(*\right.$.dat) file later used for $\left(\rho_{\beta}\right)$ calculation.

Table 1. Coefficient of anisotropy $(\lambda)$ and mean geometric resistivity $\rho_{m}$ for anisotropic model (Figure 11).

\begin{tabular}{lllll}
\hline $\mathbf{a}(\mathbf{m})$ & $\boldsymbol{\rho}_{\boldsymbol{\alpha}}(\mathbf{O h m m})$ & $\boldsymbol{\rho}_{\boldsymbol{\beta}}(\mathbf{O h m m})$ & $\boldsymbol{\rho}_{\mathbf{m}}=\sqrt{\rho_{\alpha} \mathbf{x} \boldsymbol{\rho}_{\boldsymbol{\beta}}}$ & $\lambda=\sqrt{\rho_{\boldsymbol{\alpha}} / \boldsymbol{\rho}_{\boldsymbol{\beta}}(\boldsymbol{\%})}$ \\
\hline 1 & 35.50 & 34.83 & 35.163 & 1.01 \\
3 & 29.80 & 23.00 & 26.180 & 1.14 \\
5 & 28.91 & 18.23 & 22.96 & 1.26 \\
7 & 28.19 & 14.24 & 20.04 & 1.41 \\
\hline
\end{tabular}

Furthermore it is possible to calculate the coefficient of anisotropy $(\lambda)$ and mean geometric resistivity $\left(\rho_{\mathrm{m}}\right)$. Since the 1D anisotropic model includes the thin covering isotropic layer it affects square array resistivity measurements. With square array side $a=3$ meters the anisotropy coefficient calculated in this way is $\lambda=\sqrt{ } \rho_{\alpha} / \rho_{\beta}=1.138$ (Table 1.). For smaller size of the square array side $(\mathrm{a}=1 \mathrm{~m})$ the anisotropy coefficient is only 1.01 , but its value increases with increasing value of (a), taking values of $1.26(\mathrm{a}=5 \mathrm{~m})$ and $1.41(\mathrm{a}=7 \mathrm{~m})$.

\subsection{D Forward and Inverse Modelling of the Three Layer ID Anisotropic Model}

The three layer 1D anisotropic model is shown in Figure 20, with 6.4 meters thick middle anisotropic layer (consisting of 0.5 meters thin vertical layers with varying resistivity of 10 and 300 Ohmm, respectively), while covering layer (1.1 meter thick, $50 \mathrm{Ohmm})$ and the third layer (3000 Ohmm) are homogenous and isotropic.

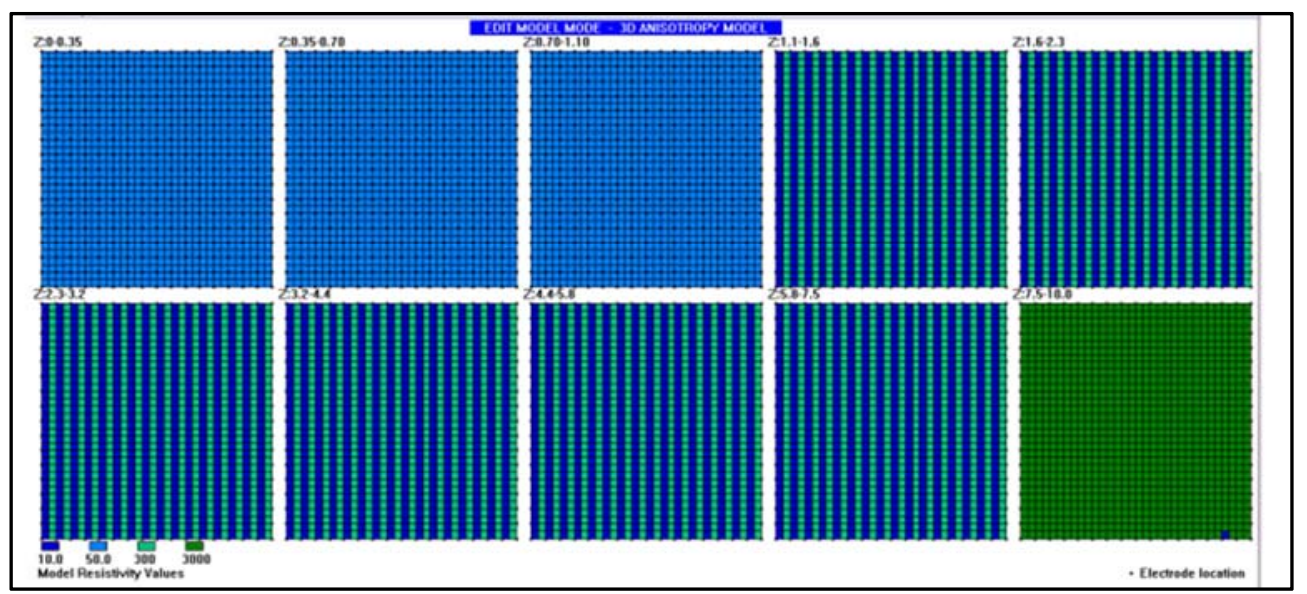

Figure 20. The three layer 1D anisotropic model (17x17 electrode grid). 


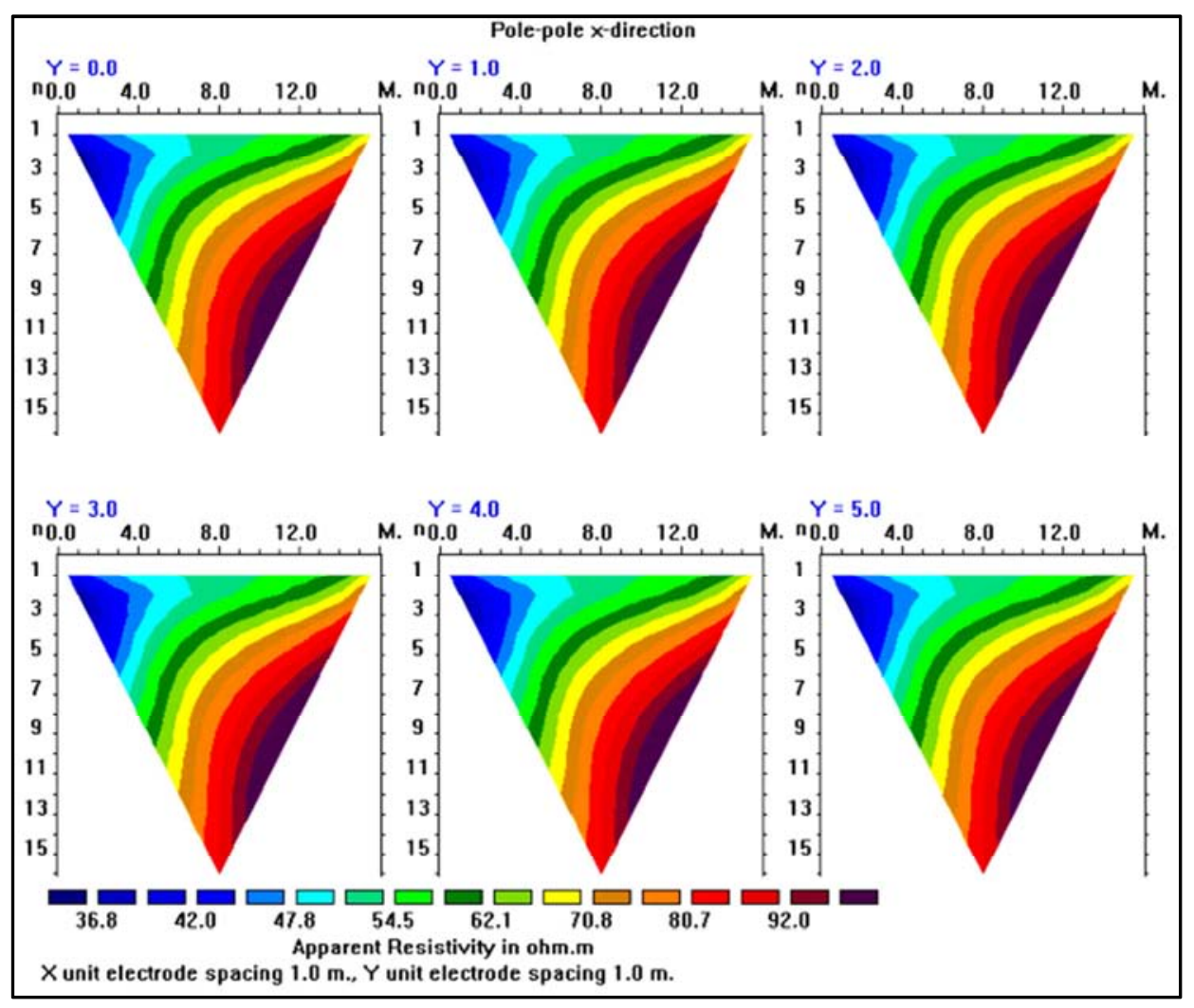

Figure 21. Pole-pole array apparent resistivity cross-sections - X direction.

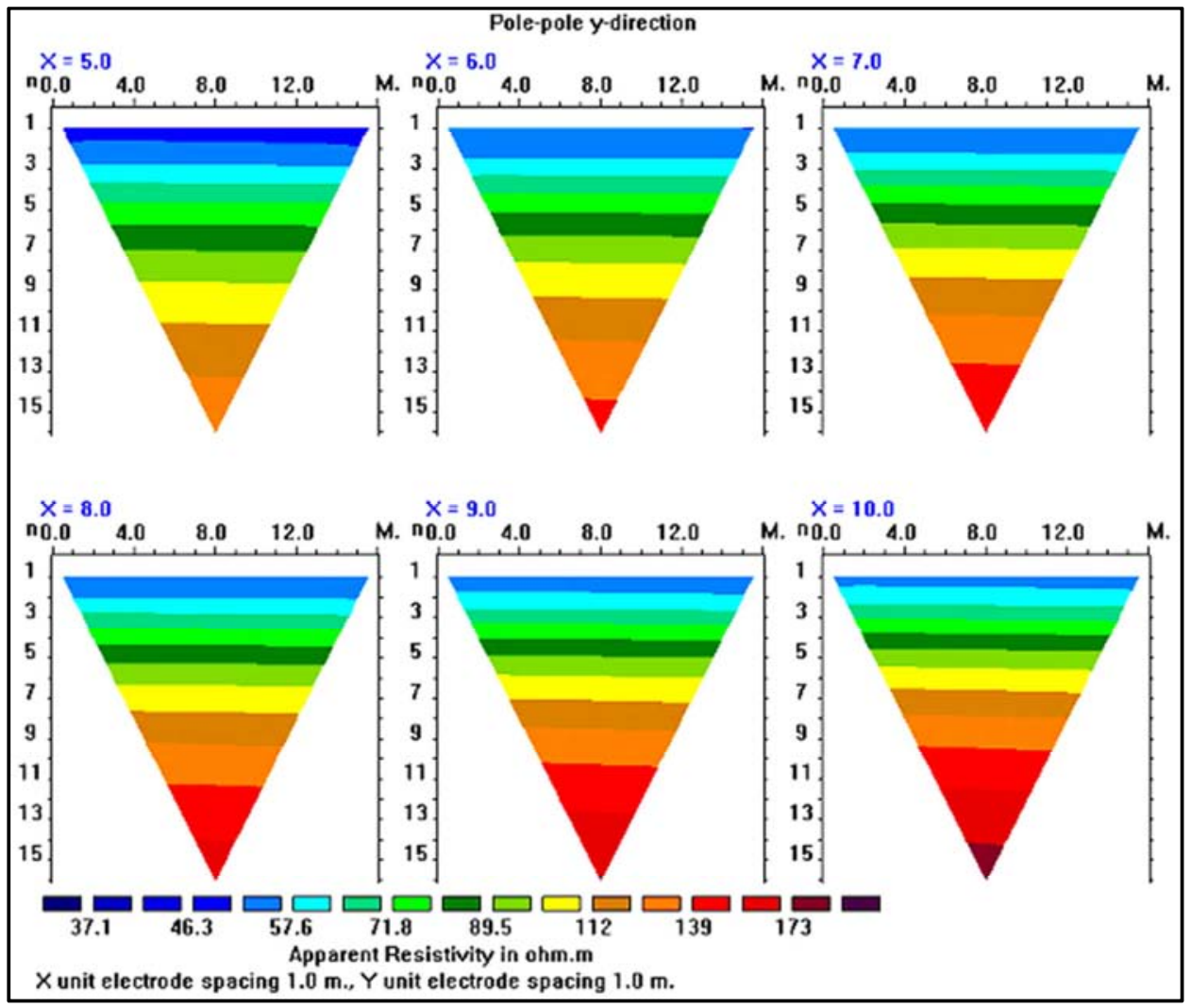

Figure 22. Pole-pole array apparent resistivity cross-sections - Y direction.

It is obvious that apparent resistivity cross-sections are both misleading (Figures 21 and 22), so that the result of 3D inversion is totally erroneous (Figure 23). 


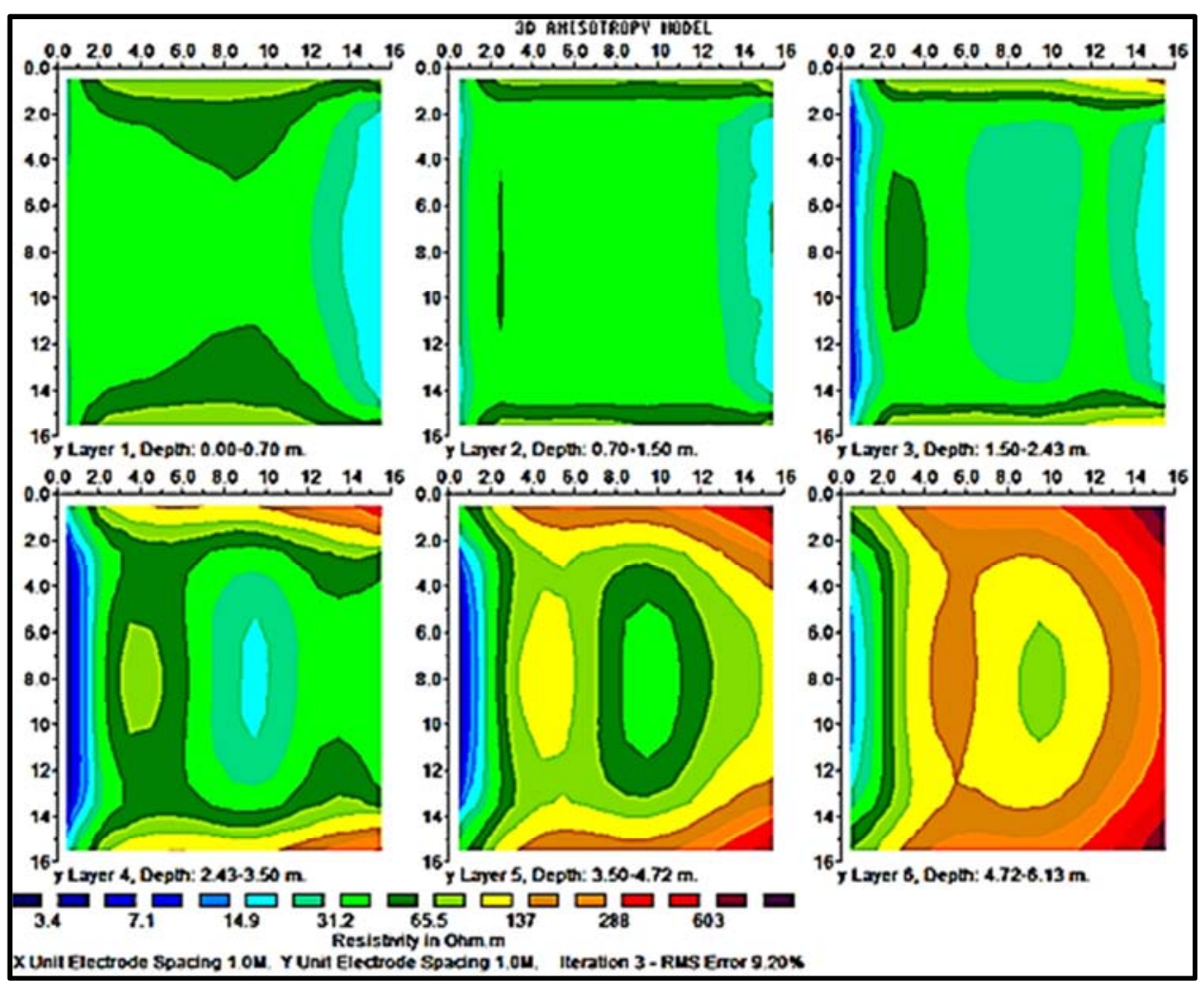

Figure 23. 3D inversion model.-horizontal sections

Square array was favourable oriented to the electrical strike, so that $\left(\rho_{\alpha}\right)$ value represents apparent resistivity measured perpendicular and $\left(\rho_{\beta}\right)$ apparent resistivity measured parallel to the strike of thin vertical layers (inside the middle layer in 1D model) which cause electrical anisotropy. The vertical layering inside the middle layer enables determination of true anisotropy coefficient $(\lambda)$ values for expanding square array dimensions and thus increasing depth of investigation (Table 2).

Table 2. Coefficient of anisotropy ( $\lambda$ ) and mean geometric resistivity $\rho_{m}$ for anisotropic model (Figure 20).

\begin{tabular}{lllll}
\hline $\mathbf{a}(\mathbf{m})$ & $\boldsymbol{\rho}_{\boldsymbol{\alpha}}(\mathbf{O h m m})$ & $\boldsymbol{\rho}_{\boldsymbol{\beta}}(\mathbf{O h m m})$ & $\boldsymbol{\rho}_{\mathrm{m}}=\sqrt{\rho_{\alpha} \mathbf{x} \boldsymbol{\rho}_{\boldsymbol{\beta}}(\mathbf{O h m m})}$ & $\boldsymbol{\lambda}=\sqrt{\rho_{\alpha} / \boldsymbol{\rho}_{\beta}(\%)}$ \\
\hline 2 & 56.9 & 55.4 & 56.1 & 1.01 \\
4 & 76.4 & 69.4 & 72.8 & 1.05 \\
6 & 95.1 & 83.0 & 88.8 & 1.07 \\
8 & 108.8 & 90.4 & 99.2 & 1.10 \\
10 & 117.6 & 95.9 & 106.2 & 1.11 \\
12 & 127.5 & 97.6 & 111.6 & 1.14 \\
14 & 133.6 & 98.9 & 114.9 & 1.16 \\
16 & 139.7 & 98.2 & 117.1 & 1.19 \\
\hline
\end{tabular}

The covering isotropic layer as well as the isotropic substratum (Figure 20) affected measured apparent resistivity $\left(\rho_{\alpha}\right)$ and $\left(\rho_{\beta}\right)$ so that $(\lambda)$ values are reduced compared to the results given in Table 1. The mean geometric apparent resistivity curve $\left(\rho_{\mathrm{m}}\right)$, which is orientation- independent, was interpreted by using $1 \mathrm{D}$ software with fixing depth to the boundaries in the three layer 1D anisotropic model (Figure 24). This square array resistivity curve was first shifted in accordance with the adequate percentage $(33 \%)$ to give an equivalent Wenner array $\left(\rho_{\mathrm{m}}\right)$ curve [12-13]. Resistivity of the relatively thin covering homogenous and isotropic layer is underestimated (40 Ohmm) due to lack of square array measurements at spacing lesser than 2 meters. On the other hand, maximal spacing of square array for $17 \times 17$ electrode grid was 16 meters so that there were not enough square array data for better defining the resistivity of half-space. Interpreted resistivity value of the middle anisotropic layer (76.5 Ohmm) is much higher than the resistivity of this layer obtained by 2D inversion (16.5-27.5 Ohmm in Figure 3) and by $1 \mathrm{D}$ inversion $(25-28.5 \mathrm{Ohmm}$ in Figures 4 and 5). This is a consequence of $1 \mathrm{D}$ inversion of orientation-independent $\rho_{\mathrm{m}}$ square array sounding curve.

Two-dimensional forward and inverse modelling was performed for Wenner array oriented perpendicularly to the electrical strike in the 1D anisotropic model in Figure 2 and as a result apparent resistivity values were minimal. Therefore interpreted resistivity value of the middle anisotropic layer is also minimal and variable with array orientation. The same is the case with 1D inversion of the Wenner sounding curve which was reconstructed from 2D Wenner cross-section. 


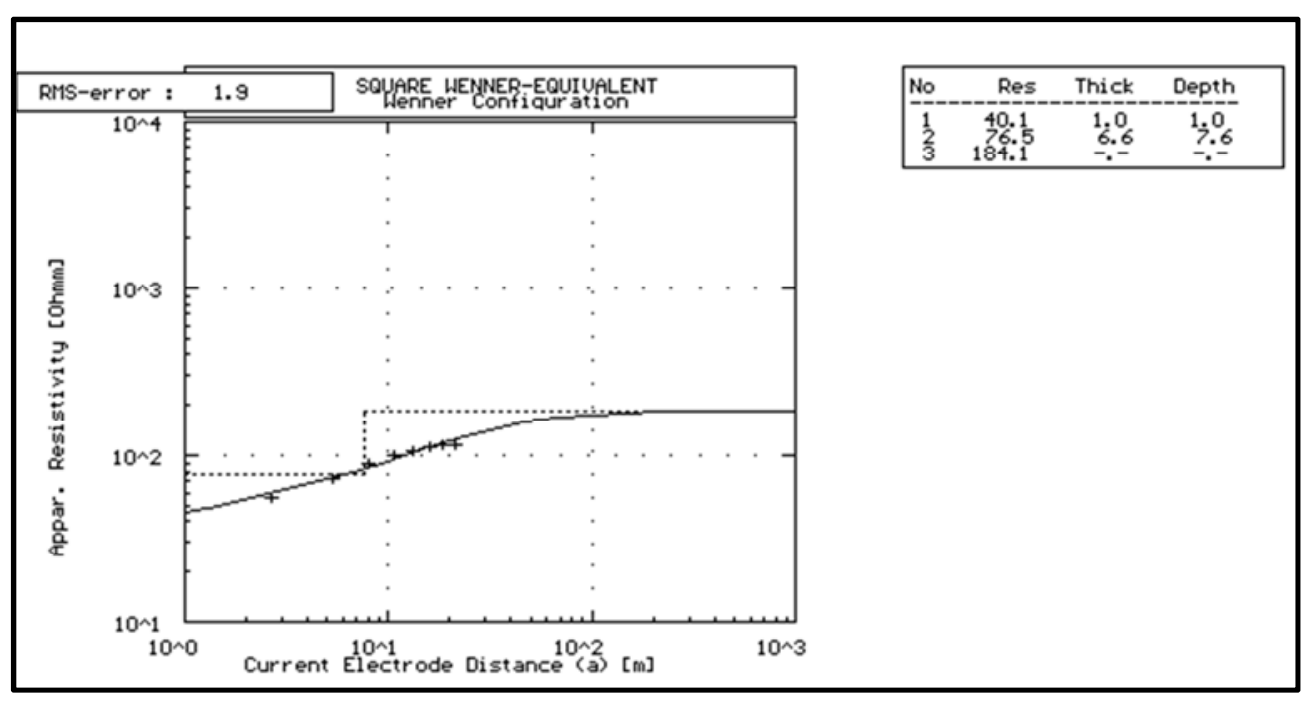

Figure 24. One dimensional interpretation of the mean geometric $\left(\rho_{m}\right)$ curve.

The square array mean geometric apparent resistivity curve $\left(\rho_{\mathrm{m}}\right)$, which is orientation-independent, is therefore the best choice for getting reliable results of 1D inversion.

\section{Conclusions}

This study is based on a number of synthetic 1D and 2D anisotropic models which were used for computing the corresponding Wenner and Pole-pole array apparent resistivity cross-sections by using forward modelling. Random noise of $\pm 2 \%$ was added to the each computed apparent resistivity data set in order to simulate measured apparent resistivity data. Apparent resistivity data which were obtained in this way served as an input for $1 \mathrm{D}, 2 \mathrm{D}$ and $3 \mathrm{D}$ inversions in order to get the idea about the effects of electrical anisotropy on the results of these inversions.

The three layer 1D anisotropic model was first considered. The relatively thick middle layer characterized by an internal oblique stratification with high resistivity contrast of 30:1 between alternating thin oblique layers was anisotropic while covering layer and substratum were homogeneous and isotropic. The result of 2D inversion of the Wenner apparent resistivity data corresponding to the $1 \mathrm{D}$ three layer anisotropic model showed that the interpreted depth to the substratum was underestimated by at least $15-20 \%$, and that the middle layer appears to be isotropic with relatively low "true" resistivity (16.5-27.5 Ohmm). This leads to completely wrong geophysical as well as geological interpretation of 2D inverse model. Further problem in interpreting apparent resistivity data acquired with any collinear array arises from the fact that these data are orientation- dependent in the presence of electrical anisotropy.

Since 2D interpretation is somewhat limited by the fact that there are no sharp boundaries between layers it was necessary to use $1 \mathrm{D}$ resistivity sounding data inversion. The 1D anisotropic model was further examined by 1D interpretation of Wenner apparent resistivity sounding curve reconstructed from Wenner array 2D cross-section. In order to better estimate the "true" resistivity of the middle anisotropic layer this 1D inversion was performed by fixing all known parameters of the model, namely thicknesses of layers and resistivities of covering layer and half-space. The "true" resistivity of the middle layer determined by this 1D interpretation was $28.5 \mathrm{Ohmm}$. The range of equivalence was also examined by keeping the RMS error value around $2 \%$ and by allowing changing of the middle layer resistivity, the first layer parameters and the resistivity of half-space being fixed. Further lowering of the resistivity of the middle anisotropic layer was possible to the value of $25 \mathrm{Ohmm}$. As a consequence the thickness of the middle anisotropic layer was decreased from 32.5 to about 27 meters and the depth to substratum from 35 to 29.4 meters, which is closer to the results of 2D inversion.

The 2D anisotropic model was composed of the same three layers as in $1 \mathrm{D}$ anisotropic model, the middle layer had the same internal structure consisting of thin alternating oblique layers which cause electrical anisotropy. In addition, this model is characterized by the trench structure in the basement which can cause structural anisotropy as well. The results of $2 \mathrm{D}$ inversion showed underestimated depth to substratum (of about $15-20 \%$ ) as well as a relatively low resistivity of the middle anisotropic layer (14-23 Ohmm) which appears to be a homogenous and isotropic layer. This can lead to wrong geophysical and geological interpretation. On the other hand the geometry of the trench-like structure was pretty well defined.

In order to investigate $3 \mathrm{D}$ forward and inverse modelling ten parallel 2D Wenner array profiles were created with the same middle anisotropic layer present in 2D models and with the same covering layer and basement parameters. The depth to the basement and to the bottom of the trench is getting shallower from profile 1 towards profile 10, while the trench structure is getting narrower in the same direction. Threedimensional inversion based on 2D inversion of apparent resistivity data sets along ten parallel profiles ( 5 meters apart) revealed that the anisotropic layer filling up the $2 \mathrm{D}$ trench structure shows very low "true" resistivity values (7.5 to 15 Ohmm), which is much lower than its values based on 1D 
and $2 \mathrm{D}$ inversions. The covering layer ( 5 meters thick and with resistivity of $50 \mathrm{Ohmm}$ ) is generally well defined as well as the trench structure which gets wider and deeper from profile 10 towards profile 1 .

For 1D anisotropic two-layer model, consisting of the thin $(0.7 \mathrm{~m})$ homogenous and isotropic $(50 \mathrm{Ohmm})$ covering layer and anisotropic half-space with vertical thin layers $(0.5 \mathrm{~m})$ with alternating resistivity values (10 and $300 \mathrm{Ohmm})$, the Pole-pole apparent resistivity 3D data set was calculated and random noise of $\pm 2 \%$ was added to simulate Pole-pole measured data set. The results of $3 \mathrm{D}$ inversion of the complete Pole-pole data sets in the case of this simple 1D anisotropic model showed totally erroneous results regarding geometry of the 3D inverse model and resistivity distribution in the $3 \mathrm{D}$ inverse model.

The same was the case regarding the three layer 1D anisotropic model which consists of 1.1 meter thick homogenous and isotropic covering layer (50 Ohmm), 6.4 meters thick anisotropic middle layer consisting of vertical thin $(0.5 \mathrm{~m})$ layers with alternating resistivity values (10 and $300 \mathrm{Ohmm})$ and homogenous and isotropic half-space (3000 Ohmm). In order to get some information on electrical anisotropy which caused totally erroneous $3 \mathrm{D}$ inverse models even for the simple 1D anisotropic models it is possible to use the complete 3D measured Pole-pole array data sets. This kind of 3D measurements enable the reconstruction of square array data sets which can further be used for electrical anisotropy analysis and for getting orientation-independent apparent resistivity sounding curves $\left(\rho_{\mathrm{m}}\right)$. Square array $\left(\rho_{\alpha}\right)$ and $\left(\rho_{\beta}\right)$ curves were calculated by using the four adequate Pole-pole array resistivity values. Square array was favorable oriented to the strike of vertical thin layers with alternating resistivity values (10 and $300 \mathrm{Ohmm}$ ), so that $\left(\rho_{\alpha}\right)$ value represents apparent resistivity measured perpendicular to the electrical strike and $\left(\rho_{\beta}\right)$ apparent resistivity measured parallel to the electrical strike. For increasing square array spacing the values of true anisotropy coefficients $(\lambda)$ were determined ranging from 1.01 to 1.19 (three layer1D anisotropic model) and from 1.01 to 1.41 (two layer1D anisotropic model).

Finally, the square array mean geometric apparent resistivity curve $\left(\rho_{\mathrm{m}}=\sqrt{ } \rho_{\alpha} \times \rho_{\beta}\right)$, which is orientationindependent, was interpreted by using 1D inversion with fixing depth to the boundaries in the three layer 1D anisotropic model. In order to interpret this square array mean geometric resistivity curve by using 1D standard software (for Wenner array) it was first necessary to shift it for the adequate percentage (33\%) to give an equivalent Wenner array mean geometric resistivity curve. The result of $1 \mathrm{D}$ inversion shows the the middle anisotropic layer is characterized by much higher "true" resistivity (76.5 Ohmm) compared to the resistivity of this layer obtained by 2D inversion of Wenner apparent resistivity cross-section (16.5$27.5 \mathrm{Ohmm}$ ) and 1D inversion of corresponding Wenner sounding curve (25-28.5 Ohmm). Two-dimensional forward and inverse modelling in this case was performed by using Wenner array oriented perpendicularly to the electrical strike, apparent resistivity values being minimal and therefore interpreted "true" resistivity value of the middle anisotropic layer is also minimal and variable with array orientation. The square array mean geometric resistivity curve $\left(\rho_{\mathrm{m}}\right)$, which is orientation-independent, is therefore the best choice for getting reliable results of $1 \mathrm{D}$ inversion but also for qualitative interpretation by using mean geometric resistivity crosssections $\left(\rho_{\mathrm{m}}\right)$, as well as $\left(\rho_{\mathrm{m}}\right)$ maps and 3D blocks of the mean geometric resistivity distribution.

\section{References}

[1] N. Inyang Udosen. N. Jimmy George, 2018, Characterization of electrical anisotropy in North Yorkshire, England using square arrays and electrical resistivity tomography Geomech. Geophys. Geo-energ. Geo-resour. July 2018 DOI: 10.1007/s40948-018-0087-5.

[2] Habberjam, G. M., Apparent resistivity, anisotropy and strike measurements. Geophysical Prospecting, 23, p. 211-247, 1975.

[3] Bhattacharya B. B., Patra H. P. Direct methods in geoelectric sounding: Principle and interpretation. Elsevier Science Publ. Co. Inc., 1968, 131 p.

[4] Loke, M. H., Tutorial: 2-D and 3-D electrical imaging surveys 1996-2018, 2018.

[5] Loke, M. H., Rapid 2-D Resistivity \& IP inversion using the least-squares method, 2008.

[6] Loke, M. H., Rapid 3D Resistivity \& IP inversion using the least-squares method, 2008.

[7] Bart Vander Velpen, RESIST. A computer program to process resistivity sounding data on PC Compatibles, Computers \& Geosciences, 19(5):691-703 - May 1993, DOI: 10.1016/00983004(93)90102-B.

[8] Koefoed. O., Geosounding Principles: Resistivity Sounding Measurements (METHODS IN GEOCHEMISTRY AND GEOPHYSICS), Elsevier Scientific Publishing Company, 1979.

[9] Habberjam G. M., Apparent resistivity observations and the use of square array techniques. (Geoexploration Monographs, Number 9), 1979. ISBN 978-3-443-13013-8.

[10] Habberjam, G. M., The effects of anisotropy on square array resistivity measurements. Geophysical Prospecting, 20, p. 249-266, 1972.

[11] Habberjam, G. M., and Watkins, G. E. The use of a square configuration in resistivity prospecting. Geophysical Prospecting, 15, p. 445-467, 1967.

[12] Lane, J. W., Jr., Haeni, F. P., and Watson, W. M., 1995, Use of square-array direct-current resistivity methods to detect fractures in crystalline bedrock in New Hampshire: Groundwater, v. 33, no. 3, p. 476-485.

[13] Geological Survey (USGS), Nye County Nuclear Waste Repository Project, 2005, Square-Array Direct-Current Resistivity Measurements Conducted at Nye County near Borehole NC-EWDP-29P. 